\title{
Unified Approach to Implicit and Explicit Solvent Simulations of Electrochemical Reaction Energetics
}

Joseph Gauthier, Colin Dickens, Hendrik H. Heenen, Stefan Ringe, Karen Chan

Submitted date: 01/07/2019 - Posted date: 01/07/2019

Licence: CC BY-NC-ND 4.0

Citation information: Gauthier, Joseph; Dickens, Colin; Heenen, Hendrik H.; Ringe, Stefan; Chan, Karen (2019): Unified Approach to Implicit and Explicit Solvent Simulations of Electrochemical Reaction Energetics. ChemRxiv. Preprint.

One of the major open challenges in ab initio simulations of the electrochemical interface is the determination of electrochemical barriers under a constant driving force. Existing methods to do so include extrapolation techniques based on fully explicit treatments of the electrolyte, as well as implicit solvent models which allow for a continuous variation in electrolyte charge. Emerging hybrid continuum models have the potential to revolutionize the field, since they account for the electrolyte with little computational cost while retaining some explicit electrolyte, representing a "best of both worlds" method. In this work, we present a unified approach to determine reaction energetics from both fully explicit, implicit, and hybrid treatments of the electrolyte based on a new multi-capacitor model of the electrochemical interface. A given electrode potential can be achieved by a variety of interfacial structures; a crucial insight from this work is that the effective surface charge gives the true driving force of electrochemical processes. In contrast, we show that the traditionally considered work function gives rise to multi-valued functions depending on the simulation cell size. Furthermore, we show that the reaction energetics are largely insensitive to the countercharge distribution chosen in hybrid implicit/explicit models, which means that any of the myriad implicit electrolyte models can be equivalently applied. This work thus paves the way for the accurate treatment of ab initio reaction energetics of general surface electrochemical processes using both implicit and explicit electrolyte.

File list (2)

main_text.pdf (3.04 MiB)

view on ChemRxiv - download file supp_info.pdf $(525.71 \mathrm{KiB})$ view on ChemRxiv • download file 


\title{
Unified approach to implicit and explicit solvent simulations of electrochemical reaction energetics
}

\author{
Joseph A. Gauthier,,$+\neq$ Colin F. Dickens, ${ }^{\dagger, \ddagger}$ Hendrik H. Heenen, "Stefan Ringe, \\ and Karen Chan*, \\ $\dagger$ †UNCAT Center for Interface Science and Catalysis, Department of Chemical \\ Engineering, Stanford University, Stanford, California 94305, United States \\ $\ddagger S U N C A T$ Center for Interface Science and Catalysis, SLAC National Accelerator \\ Laboratory, 2575 Sand Hill Road, Menlo Park, California 94025, United States \\ IDepartment of Physics, Technical University of Denmark, DK-2800, Kgs. Lyngby, \\ Denmark \\ E-mail: kchan@fysik.dtu.dk
}

\begin{abstract}
One of the major open challenges in ab initio simulations of the electrochemical interface is the determination of electrochemical barriers under a constant driving force. Existing methods to do so include extrapolation techniques based on fully explicit treatments of the electrolyte, as well as implicit solvent models which allow for a continuous variation in electrolyte charge. Emerging hybrid continuum models have the potential to revolutionize the field, since they account for the electrolyte with little computational cost while retaining some explicit electrolyte, representing a "best of both worlds" method. In this work, we present a unified approach to determine reaction energetics from both fully explicit, implicit, and hybrid treatments of the electrolyte based on a new multi-capacitor model of the electrochemical interface. A
\end{abstract}


given electrode potential can be achieved by a variety of interfacial structures; a crucial insight from this work is that the effective surface charge gives the true driving force of electrochemical processes. In contrast, we show that the traditionally considered work function gives rise to multi-valued functions depending on the simulation cell size. Furthermore, we show that the reaction energetics are largely insensitive to the countercharge distribution chosen in hybrid implicit/explicit models, which means that any of the myriad implicit electrolyte models can be equivalently applied. This work thus paves the way for the accurate treatment of ab initio reaction energetics of general surface electrochemical processes using both implicit and explicit electrolyte.

\section{Introduction}

Computational chemistry tools developed over the past several decades have allowed for an unprecedented level of mechanistic understanding in a wide variety of interface phenomena. ${ }^{1-22}$ In particular, electrocatalysis has experienced a rapid expansion with the application of density functional theory (DFT). The computational hydrogen electrode model ${ }^{23}$ has, in the past 15 years, enabled the determination of the thermochemistry of coupled ion-electron transfers using simple surface science calculations. For a number of applications, however, electrochemical reaction barriers, ${ }^{24-37}$ electric field, ${ }^{38-40}$ and $\mathrm{pH}$ effects, ${ }^{41-43}$ are important, and all these elements require careful consideration of the electrolyte. Several methods have since been developed to model the electrochemical interface in ab initio simulations. Fully explicit simulations of the electrolyte ${ }^{44-46}$ have been developed, and in parallel, different implicit solvation methods have been implemented in a variety of commonly used DFT codes. $^{17,47-64}$

Each of these techniques comes with their own advantages and shortcomings. Fully explicit techniques give atomistic insight to the effects of solvation and the effect of electric field on reaction energetics. ${ }^{13,40,44}$ However, they frequently rely on expensive electrolyte sampling methods, ${ }^{11,65-67}$ and furthermore suffer from issues related to band misalignment, ${ }^{68}$ stem- 
ming from the well known failure of GGA-DFT under-predicting bandgaps. ${ }^{69}$ Continuum methods present thermodynamic averages of solvation and therefore do not rely on expensive sampling techniques. They do however come with a variety of challenges ${ }^{70}$ primarily from the marrying of the classical treatment of solvation with the atomistic treatment of the surface. Most importantly, continuum methods intrinsically cannot reproduce the chemical interaction of the surface with ions, since the charge is necessarily smeared over the entire electrode surface. Solvation energies for these methods have primarily been fit to molecular solutes, since that is the source of most experimental reference data. ${ }^{47,54,56,71}$ Little work has been done to benchmark these energies on extended surfaces, where hydrogen bonding has been shown to be important. ${ }^{25,72-74}$ For these reasons, hybrid approaches, including both some explicit electrolyte molecules and continuum solvation, are receiving a growing amount of attention. ${ }^{75,76}$ 

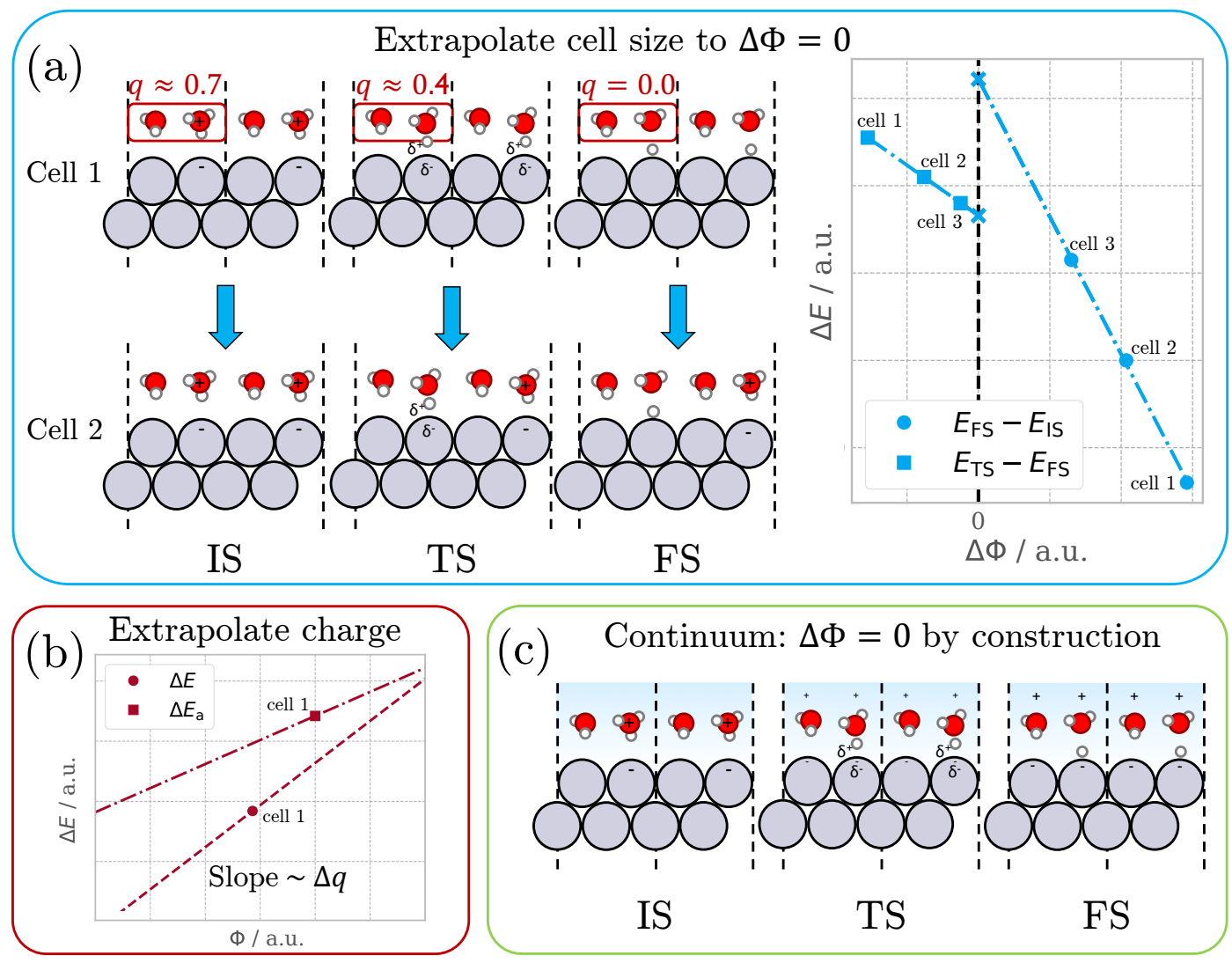

Figure 1: Schematic of three current state of the art methods for calculating reaction energetics at constant driving force. (a) Schematic of cell extrapolation for two cell sizes, and illustration of charge partitioning (e.g. Bader) to determine the charge of the ion at the interface for charge extrapolation. (b) Charge extrapolation technique, with charges determined by partitioning as illustrated in (a). (c) Continuum charging, which exchanges electrons with an external reservoir to achieve constant work function. Counter charge is placed in the electrolyte to retain overall charge neutrality. 
Real electrochemical systems operate at a constant applied electrode potential, and so there has been an effort to develop computational models that allow for simulations at constant potential. This is particularly challenging, since the work function, which defines the potential in ab initio simulations, changes across the reaction coordinate due the use of finite simulation cell sizes. Figure 1 illustrates three classes of techniques used currently to solve the problem of changing work function across the reaction coordinate during charge transfer reaction events. Cell extrapolation, illustrated in Figure 1(a), relies on systematically increasing the size of the simulation cell in order to more closely approximate the infinite cell size limit. This approach can be quite expensive computationally, owing to the roughly cubic scaling of DFT with system size. ${ }^{77}$ Charge extrapolation, illustrated in Figure 1(b), instead utilizes a mean-field (i.e. capacitor) approximation of the interface to predict the slope of the energy as a function of potential, which is proportional to the change in charge between states. The primary difficulty with this method is identifying the charge in each state. Previous work has shown that the charge resulting from charge partitioning schemes (e.g. Bader ${ }^{78-80}$ ) can accurately reproduce the results from cell extrapolation, ${ }^{45,46}$ but it is not clear how to partition charge for field-sensitive chemical steps. Finally, continuum charging methods, shown in Figure 1(c), allows for continuous variation in charge, leading to the possibility of the work function being constant between states at little additional computational cost. $^{75}$

In the present work, we present a unified approach to determining reaction energetics for general hybrid or fully explicit electrolyte models of the interface. This framework is based on 1) a multi-capacitor model of the various components of an electrochemical interface in atomistic DFT simulations 2) the idea that an effective surface charge density is the appropriate descriptor for the electrochemical driving force of electrochemical reactions, as opposed to the generally applied work function. The latter is essentially a reformulation of the Frumkin correction to reaction kinetics, ${ }^{81,82}$ which emphasizes that the local potential drop at the interface between the metal and reaction plane potential is the driving force 
for electrochemical reactions. What we assert here is that this local potential drop is best approximated by an effective surface charge density. In contrast, the traditional use of work function as a descriptor of the driving force leads to multi-valued, cell-size dependent functions of reaction energetics in hybrid implicit/explicit simulations. We further show that, within our framework, the reaction energetics are essentially insensitive to the distribution of the continuum countercharge, which means that any of the myriad of existing implicit electrolyte models can be equivalently applied. By using the effective surface charge as the descriptor for the driving force, we importantly avoid the need to parameterize the continuum model to accurately predict capacitances, for which experimental data can be lacking. The capacitances also vary significantly upon the addition of explicit water layer(s) or at significant coverages of certain adsorbates, which renders parametrizations to bare surfaces obsolete. The energetics obtained through our method as a function of surface charge can be related to experimental activities through a coupled double-layer charging kinetic model, where diffuse layer effects can be accounted for. The framework we present here solves the observed cell-size dependence in hybrid explicit-implicit solvation constant potential simulations, and therefore represents a major step forward in the computational modeling of electrochemical reaction energetics.

\section{Theoretical Methods and Models}

This work uses the Vienna ab-initio Software Package ${ }^{83-85}$ in conjunction with VASPsol, ${ }^{56,57}$ as well as QUANTUM-ESPRESSO ${ }^{86}$ (QE) in conjunction with the Environ ${ }^{47}$ solvation module. Using VASP, core electrons were modeled with projector augmented wave pseudopotentials; using QE, core electrons were modeled using GBRV ultrasoft pseudpotentials. ${ }^{87}$ In both DFT codes, valence electrons were expanded as plane-waves up to a kinetic energy cutoff of $500 \mathrm{eV}$. Exchange and correlation interactions were accounted for using the RPBE functional $^{88}$ in both codes. When optimizing bulk platinum to determine the appropriate 
lattice constant, the Brillouin zone was sampled with a 12 x 12 x 12 -centered MonkhorstPack $^{89}$ k-point mesh. The optimized Pt lattice constant was determined to be $3.990 \AA$ in VASP, and the optimized $\mathrm{Cu}$ lattice constant was found to be $3.6 \AA$ in $\mathrm{QE}$.

Geometries were considered optimized when the maximum force on any unconstrained atom in the system was below $0.03 \mathrm{eV}^{-1}$. For each electronic self-consistent field calculation, the density was considered to have converged when the total energy changed by less than $10^{-4} \mathrm{eV}$ between steps. Separation between periodic images was set to be $8 \AA$. Using our previously published methodology, ${ }^{90}$ we have corrected the constant charge reaction energetics by $q \Delta \Phi_{\mathrm{vac}}$, where $q$ is the (implicit) system charge and $\Phi_{\mathrm{vac}}$ is the potential of the electrolyte region. This accounts for the shift in potential necessary with VASPsol and eliminates cell height dependence of the energetics. To avoid possible spurious dipole interactions, our simulation cells are symmetric along the direction normal to the surface.

VASPsol and Environ both treat the electrolyte at the electrochemical interface as a polarizable continuum. VASPsol places counter-charge via the linearized Poisson-Boltzmann equation, while Environ allows for a variety of countercharge placement models. In this work, except when stated otherwise, we use VASPsol, which places countercharge by solving the linearized Poisson-Boltmzann equation. Within this model we choose a Debye length of 3.0 $\AA$, corresponding to a bulk ion concentration of $1.0 \mathrm{M}$. We set the effective surface tension to zero to avoid numerical instabilities, as reported in a recent perspective. ${ }^{70}$ When Environ is used, we use the planar countercharge, modified Poisson-Boltzmann, and linearized modified Poisson-Boltzmann countercharge placement models. We use the standard g03-SCCS solvation parameters. ${ }^{47}$ Details regarding the implementation of these solvation models can be found in their documentation. ${ }^{47,56,57}$ 


\section{Results and Discussion}

We consider different kinds of charged interfacial systems as visualized in Figure 2: adsorbed partially negatively charged $\mathrm{CO}_{2}$, explicitly modeled hydronium cations and a continuum representation of electrolyte counter ions at the outer Helmholtz plane. All of these systems give rise to a separation of negative and positive charge, which can be effectively envisioned as local capacitors. In the case of explicit (e.g. proton) charge, the effective capacitor charge is just that of the proton itself, which is slightly less than unity. ${ }^{91}$ In the case of $\mathrm{CO}_{2}$, partial charge, corresponding to the electrosorption valency, is transferred during the adsorption process which leads to the creation of a strong dipole moment. ${ }^{92-94}$

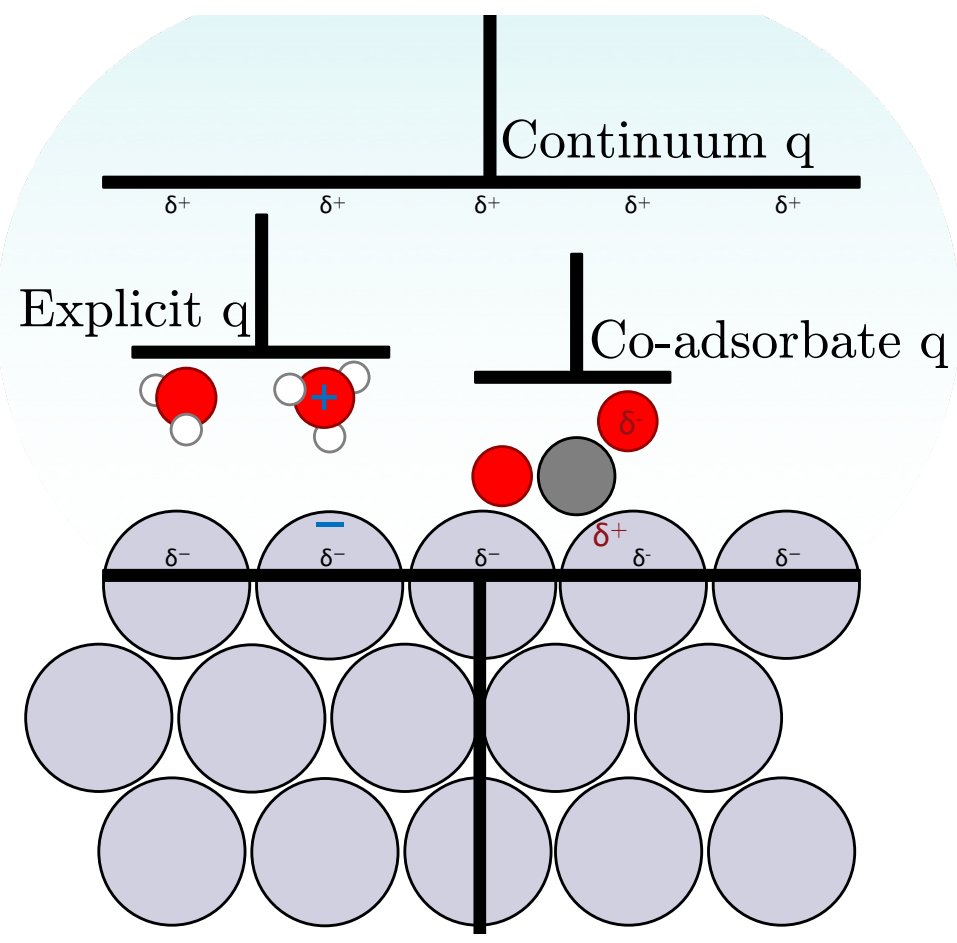

Figure 2: Illustration of three different potential dependent processes that can be effectively modeled by adiabatic DFT approaches: $\mathrm{CO}_{2}$ adsorption, charge transfer reactions (e.g. $\left.\left(\mathrm{H}^{+}+\mathrm{e}^{-}\right)+* \rightarrow \mathrm{H} *\right)$, and continuum charging/counter-charging.

The local potential drop at the interface, between the metal potential and the solution potential at the reaction plane, is the driving force for electrochemical reactions. This is the essential idea behind the Frumkin diffuse layer correction to reaction kinetics. ${ }^{81,82}$ As we 
show below, this local potential drop is better approximated by the total effective surface charge than the overall work function. This concept is illustrated in Figure 3, where the potential drop across the electrochemical interface is illustrated. Here the work function is given by the potential difference between the metal surface and the vacuum level, $\Phi_{\mathrm{M}}-\Phi_{\mathrm{vac}}$. However, the driving force for charge transfer is instead described by $\Phi_{M}-\Phi_{R P}$, since the transition state lies in this region.

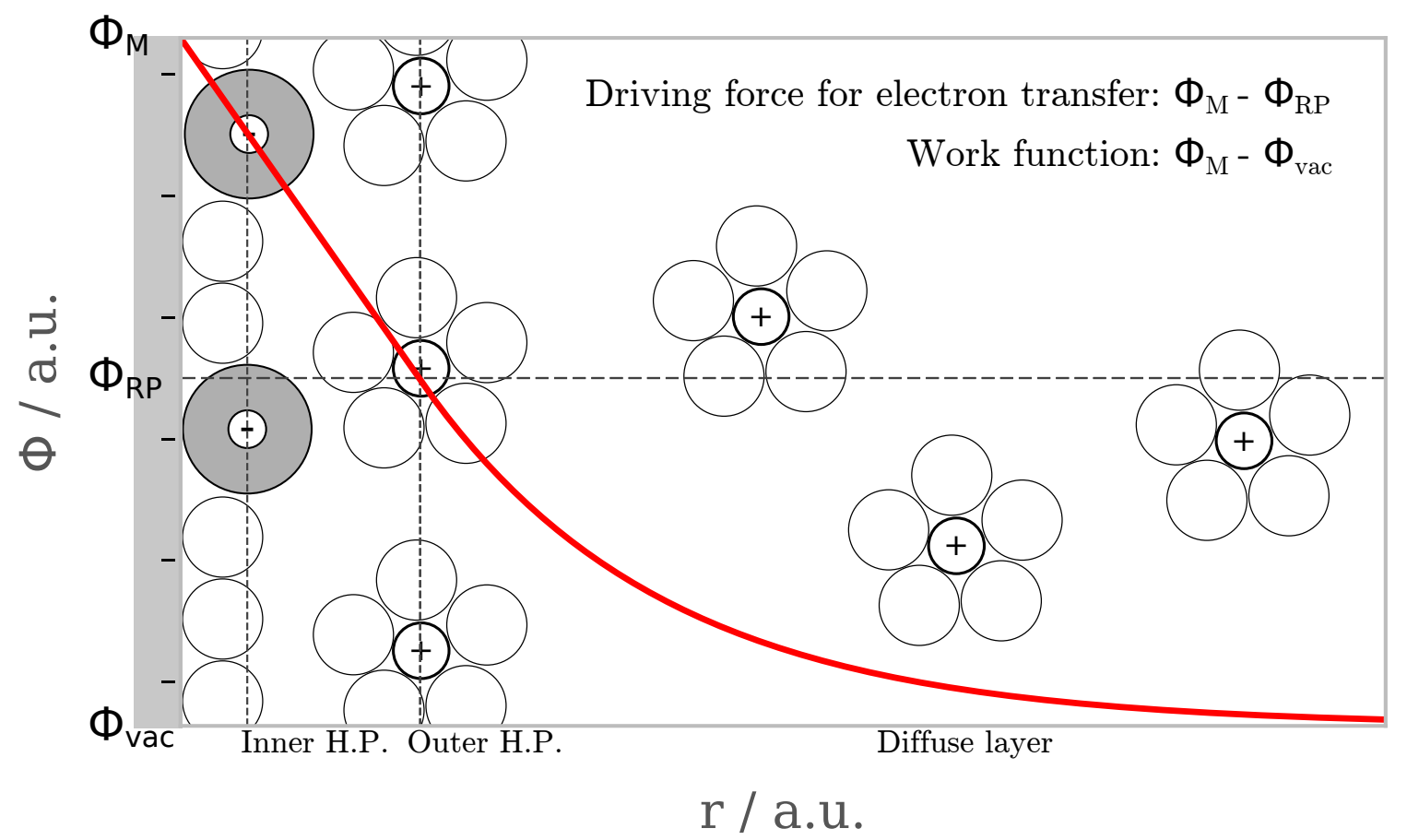

Figure 3: Illustration of the potential drop across the electrochemical interface. Here the surface (drawn as the left side of the plot), is covered by charge neutral solvent molecules (solid white circles) and anions (dark gray circles). Solvated cations form a Helmholtz like layer demarcating the outer Helmholtz plane, followed by the diffuse layer. The linear potential drop between the surface and the reaction plane gives the driving force for electron transfer $\left(\Phi_{\mathrm{M}}-\Phi_{\mathrm{RP}}\right)$, while the work function is given by potential drop between the surface and the vacuum potential $\left(\Phi_{\mathrm{M}}-\Phi_{\mathrm{vac}}\right)$.

In what follows, we derive a generic multi-capacitor model of the electrochemical interface, ${ }^{17,45,46,95-98}$ starting with the simple case of an interface with one charging component. We use this model to develop expressions relating electrochemical reaction energetics to both excess surface charge density, and the work function. We then show the "effective" surface 
charge density to be the appropriate descriptor of the driving force, since it reflects the local potential drop. We demonstrate that, using the work function (a measure of the applied potential) as the descriptor, the reaction energetics are not uniquely defined. We then develop a framework to calculate electrochemical reaction energetics in general.

\section{General Multi-Capacitor Model of the Electrochemical Interface}

\section{Energy of the Charged Interface}

We first review the energy of the charged interface undergoing only one charging process. We can expand the energy as a Taylor expansion about a hypothetical zero interfacial charge $q=0$, corresponding to a work function $\Phi_{0}$, as has been done in previous works. ${ }^{17,95-98}$

$$
E=E^{0}+\left.\frac{\partial E}{\partial q}\right|_{q=0} q+\left.\frac{1}{2} \frac{\partial^{2} E}{\partial q^{2}}\right|_{q=0} q^{2}+\mathcal{O}\left(q^{3}\right)
$$

Here $E^{0}=\left.E\right|_{q=0}$ is the energy of all non-electrostatic (i.e. chemical) components of the system at the hypothetical $q=0$ case, which corresponds to the case of zero partial charges in Figure 2. This case is exemplified by, for instance, a hypothetical neutral $\mathrm{H}_{3} \mathrm{O}$ molecule solvated above a metal slab (as opposed to a positively charged $\mathrm{H}_{3} \mathrm{O}$ molecule solvated above a negatively charged metal slab), or a hypothetical adsorbed $\mathrm{CO}_{2}$ molecule with no dipole moment.

By definition,

$$
\left.\frac{\partial E}{\partial q}\right|_{q=0}=\Phi_{0}
$$

where $\Phi_{0}$ is the work function at zero charge, referenced to the bulk electrolyte potential. We further assume here that we have a constant capacitance $C$, which we find to be valid in small potential ranges, as discussed later in this work:

$$
\frac{\partial}{\partial q}\left(\frac{\partial E}{\partial q}\right)=\frac{\partial \Phi}{\partial q}=\frac{1}{C}
$$


such that (1) becomes.

$$
E=E^{0}+q \Phi_{0}+\frac{q^{2}}{2 C}
$$

We now use the capacitor equation $q=C\left(\Phi-\Phi_{0}\right)$ to write (1) in terms of $\Phi$

$$
E=E^{0}+C\left(\Phi-\Phi_{0}\right) \Phi_{0}+\frac{C\left(\Phi-\Phi_{0}\right)^{2}}{2} .
$$

Or without reference to the capacitance,

$$
E=E^{0}+q \Phi_{0}+\frac{q\left(\Phi-\Phi_{0}\right)}{2} .
$$

As an example, we show the energetics of Pt (111) as a function of both excess surface charge $q$ as well as the work function $\Phi$ in Figure 4 . Here the surface was charged using the linearized-Poisson Boltzmann equation to place countercharge. The slight deviations from the model in panel (b) results from the capacitance being non-linear far from the potential of zero charge. From a linear fit of the surface charge as a function of potential, we calculate a surface capacitance $C$ of $25 \mu \mathrm{F} \mathrm{cm}^{-2}$, and a potential of zero charge $\Phi_{0}$ of $5.4 \mathrm{eV}$. 

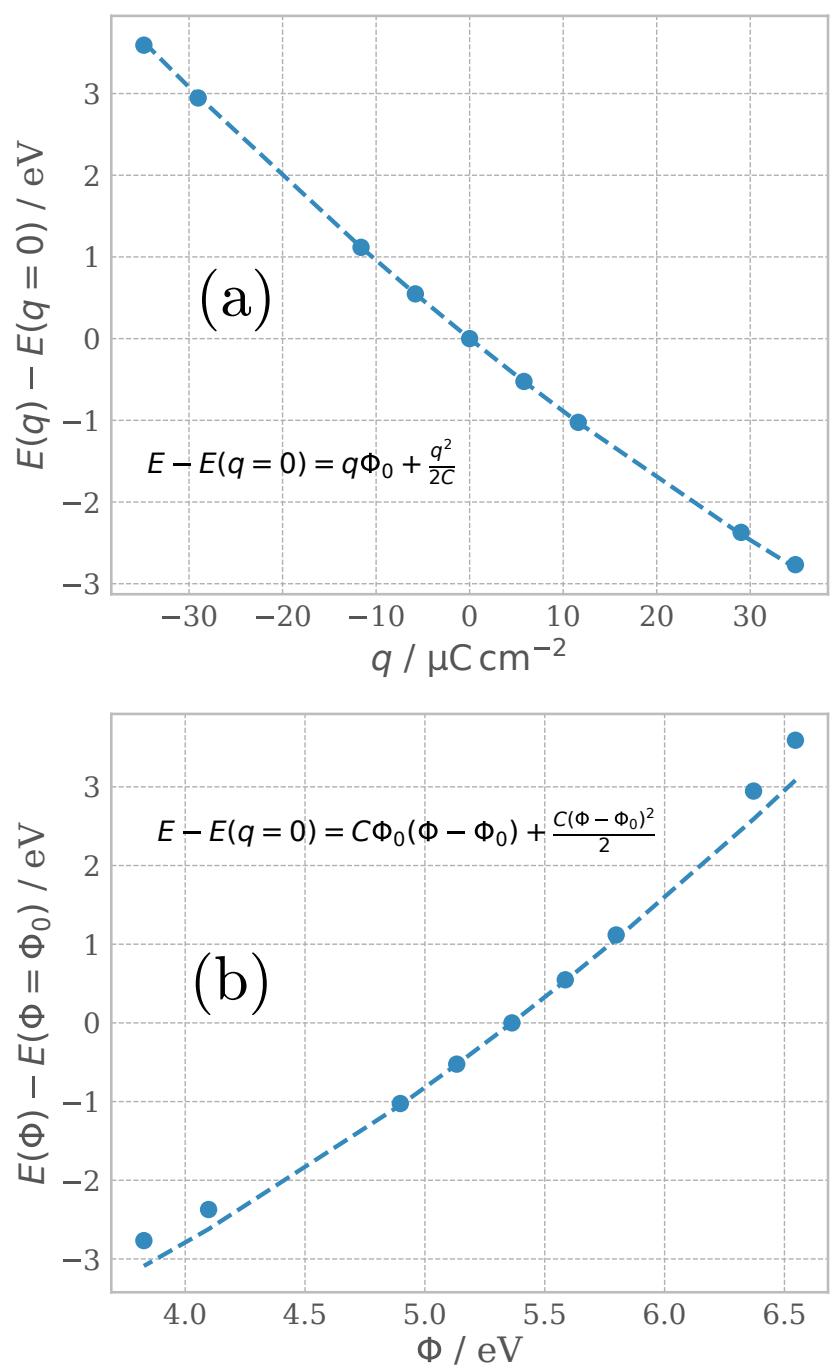

Figure 4: Energetics of $\operatorname{Pt}(111)$ as a function (a) excess implicit surface charge $q$, where dashed lines correspond to Eq. (4), and (b) work function $\Phi$, where dashed lines correspond to Eq. (6). Panel (b) shows slight deviations far from the potential of zero charge $(\approx 5.4 \mathrm{eV}$ in this picture) due to the nonlinear capacitance behavior far from the PZC. 


\section{Reaction Energetics: Only One Charging Component}

We now consider the energetics of an interfacial charge-transfer reaction, i.e. charging process of any of the envisioned capacitors in Figure 2. Considering an infinitely extended system, the charging and discharging of a single capacitor does not change the work function of the metal electrode. In a typically used finite unit cell setup, however, the charge-transfer reaction critically changes the electrode work function. As before, the hypothetical $q=0$ state is one such that there is no charge separation; for example, a hypothetical neutral hydronium molecule solvated above an uncharged metal slab, or a hypothetical $\mathrm{CO}_{2}$ molecule adsorbed with no dipole moment. We can therefore assume that $\Phi_{0}$ does not change between the states considered. We find that in practice, the capacitance varies by only a very small amount for a large change in surface coverage, changing from $12.6 \mu \mathrm{F} \mathrm{cm}^{-2}$ for a clean $\mathrm{Pt}$ (111) surface to $10.1 \mu \mathrm{F} \mathrm{cm}^{-2}$ with $\mathrm{CO}_{2}$ adsorbed in a $2 \mathrm{x} 2$ supercell, the smallest cell size considered here (and hence the highest coverage). This capacitance drop reduces to just 0.1 $\mu \mathrm{F} \mathrm{cm}^{-2}$ in a $4 \times 4$ supercell, as we reported in a recent work. ${ }^{90}$ We therefore assume that the capacitance between states does not vary.

Under these assumptions, from Eq. (4), we have the change in energy from state 1 to state 2 (denoted as subscript 1 and 2 , respectively) as

$$
\begin{aligned}
\Delta E & =\Delta E_{0}+\left(q_{2}-q_{1}\right) \Phi_{0}+\frac{q_{2}^{2}-q_{1}^{2}}{2 C} \\
& =\Delta E_{0}+\left(q_{2}-q_{1}\right)\left(\frac{\bar{q}}{A \widetilde{C}}+\Phi_{0}\right),
\end{aligned}
$$

where we have defined the average charge $\bar{q}=\frac{1}{2}\left(q_{1}+q_{2}\right)$ and introduced the surface area normalized capacitance, $\widetilde{C}=C / A$. Eq. (8) can also be written as a function of work function via $q=C\left(\Phi-\Phi_{0}\right)$,

$$
\Delta E=\Delta E_{0}+\left(q_{2}-q_{1}\right) \bar{\Phi}
$$

where we have similarly defined $\bar{\Phi}=\frac{1}{2}\left(\Phi_{1}+\Phi_{2}\right)$. In the example of $\mathrm{CO}_{2}$ adsorption, $q_{2}-q_{1}$ 
yields the effective surface charge associated with the a polarized $\mathrm{CO} 2$ molecule. For the Volmer reaction, $q_{2}-q_{1}$ yields the effective surface charge transferred. Naturally, $q_{2}-q_{1}$ could also represent the charge difference via continuum charging of any surface composition.

In the limit of an infinitely sized simulation cell, the corresponding effect of the dipole shift on the potential and surface charge density is infinitesimal, i.e. $\Phi_{1} \rightarrow \Phi_{2}$ and $q_{1} / A \rightarrow q_{2} / A$, but $q_{1} \nrightarrow q_{2}$. In the infinite cell size limit, we have

$$
\begin{aligned}
\Delta E & =\Delta E_{0}+\left(q_{2}-q_{1}\right)\left(\frac{q}{A \widetilde{C}}+\Phi_{0}\right) \\
& =\Delta E_{0}+\left(q_{2}-q_{1}\right) \Phi .
\end{aligned}
$$

The effective charge $q_{2}-q_{1}$ can be determined by evaluating the reaction energetics at multiple finite cell sizes and then fitting either Eq. 8 or Eq. 9, i.e.

$$
q_{2}-q_{1}=\frac{\partial \Delta E}{\partial \bar{\Phi}}=\frac{\partial \Delta E}{\partial\left(\frac{\bar{q}}{A \widetilde{C}}+\Phi_{0}\right)}
$$

This approach is equivalent to the cell extrapolation approach for constant potential reaction energetics, detailed in Ref. 44. Further details regarding this, and the choice of average work function as the descriptor can be found in the supporting information (SI) Note 1. There we explain that the change in energy as a function of average work function is equivalent to the cell-extrapolated energetics, where the work function does not change between the initial and final state. In the case of a simple proton-electron transfer, it has been shown in previous work that $q_{2}-q_{1}$ can be usually be determined through the Bader ${ }^{78-80}$ charge of the slab and adsorbates (i.e. the charge extrapolation model). ${ }^{45,46}$

The corresponding capacitance can be determined by fitting $\Delta E$ vs $\Phi_{2}^{2}-\Phi_{1}^{2}$, since Eq. (9) can be written

$$
\Delta E=\Delta E_{0}+\frac{C}{2}\left(\Phi_{2}^{2}-\Phi_{1}^{2}\right) .
$$

We note here that in the case of an adsorption reaction event, the calculated effective trans- 
ferred charge $q_{2}-q_{1}$ is not the charge transferred upon adsorption (i.e. the electrosorption valency $^{92-94}$ ), as seen in adsorption pseudocapacitances or in cyclic voltammetry. Rather, it is the physical charge separation at the interface that contributes to the corresponding capacitance of the given process.

To illustrate these charging behaviors, we have plotted the energetics of a simple protonelectron transfer process (Volmer, i.e. $\left.\left(\mathrm{H}^{+}+\mathrm{e}^{-}\right)+* \rightarrow \mathrm{H} *\right)$ and $\mathrm{CO}_{2}$ adsorption on $\mathrm{Pt}(111$ ) as a function of the work function $\Phi$ in Figure 5. In this picture, no continuum solvation or charging is used; the different scatter points are achieved by changing the coverage of protons and $\mathrm{CO}_{2}$, respectively by changing the unit cell size.

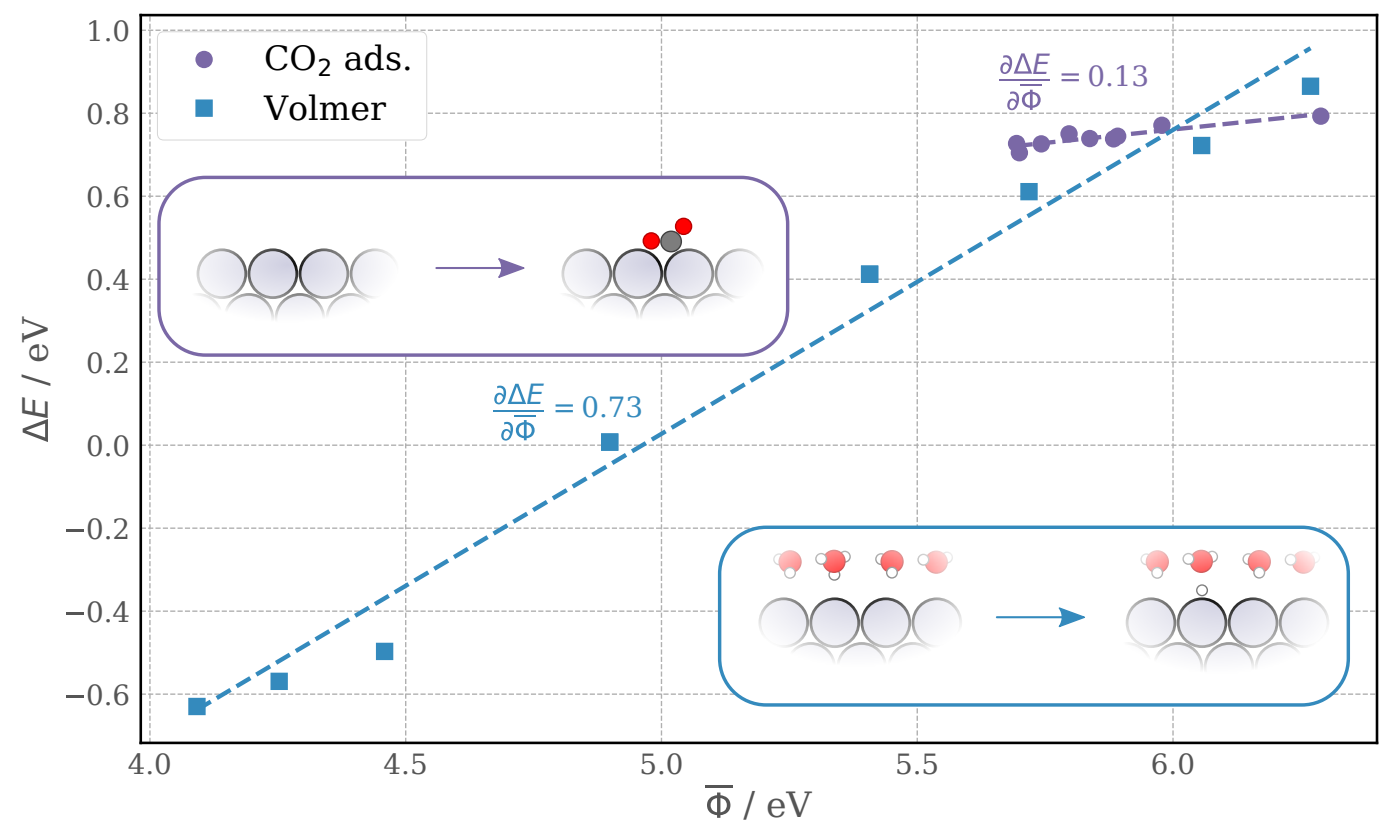

Figure 5: Energy as a function of average work function, $\bar{\Phi}=\frac{1}{2}\left(\Phi_{\mathrm{IS}}+\Phi_{\mathrm{FS}}\right)$ for $\mathrm{CO}_{2}$ adsorption on $\mathrm{Pt}(111)$ and the Volmer reaction (i.e. $\left.\left(\mathrm{H}^{+}+\mathrm{e}^{-}\right)+* \rightarrow \mathrm{H} *\right)$ on $\mathrm{Pt}(111)$, with no continuum solvation. The different scatter points are calculated by changing the coverage of $\mathrm{CO}_{2}$ and protons by, for instance, changing the cell size. Here the x-axis is the average work function between the initial and final state, and so the slopes in this plot correspond to a fit of $q_{\text {eff }}$ as given in Eq. (12). 


\section{Reaction Energetics: Two Charging Components}

We now consider the case of a reaction energy evaluated where a second charging component is present and constant throughout the reaction pathway. For example, a Volmer reaction event where a constant continuum charge $q_{\text {imp }}$ or an additional co-adsorbate with effective

charge $q_{\text {ad }}$ is added to the two states considered. As we illustrated in a previous work, ${ }^{90}$ the energetics obtained with a constant charge vs $\bar{\Phi}$ between the states considered are equivalent to the grand canonical energy difference at constant potential. The latter method of charging the interface to achieve constant potential has been applied to a wide variety of surface electrochemical reactions, ${ }^{75,99-101}$ since it allows a simple way to probe energetics across a large range of work functions.

For a general system with both implicit and explicit charging, by applying Eq. (7), we can expand the energy about $q_{\mathrm{imp}}=0$, giving a total reaction energy as the sum of contributions from both explicit and implicit charging,

$$
\begin{aligned}
\Delta E & =\Delta E_{\exp }+\Delta E_{\mathrm{imp}} \\
& =\Delta E_{\exp }+q_{\mathrm{imp}, 2} \Phi_{2}-q_{\mathrm{imp}, 1} \Phi_{1}+\frac{q_{\mathrm{imp}, 2}^{2}-q_{\mathrm{imp}, 1}^{2}}{2 C_{\mathrm{imp}}} .
\end{aligned}
$$

where $\Delta E_{\text {exp }}$, the component arising from explicit charging, is given by Eq. (8). We introduced the capacitance associated with charging the interface with a continuum charge, $C_{\mathrm{imp}}$ and note that it need not equal the capacitance associated with charging the interface via other methods. We assume that the continuum charge $q_{\text {imp }}$ is constant between initial and final state in accordance with constant charge calculations, i.e. $q_{\text {imp }, 1}=q_{\text {imp }, 2}=q_{\text {imp }}$. The 
reaction energy then reduces to

$$
\begin{aligned}
\Delta E & =\Delta E_{\exp }+q_{\text {imp }}\left(\Phi_{2}-\Phi_{1}\right) \\
& =\Delta E_{0}+\left(q_{2}-q_{1}\right)\left(\frac{\bar{q}_{\exp }}{C_{\exp }}+\Phi_{0}\right)+q_{\text {imp }}\left(\frac{q_{2}-q_{1}}{C_{\exp }}\right) \\
& =\Delta E_{0}+\frac{q_{2}-q_{1}}{\widetilde{C}_{\exp }}\left(\frac{\bar{q}_{\exp }+q_{\text {imp }}}{A}+\widetilde{C}_{\exp } \Phi_{0}\right) .
\end{aligned}
$$

\section{Effective Surface Charge Density as the Appropriate Descriptor of Driving Force}

As we noted previously, two independent charging components need not have identical associated capacitances. Here, we demonstrate that unequal capacitances lead to the average work function not uniquely defining the energetics, and that the surface charge density is a better descriptor of the local driving force.

To write Eq. (18) in terms of potential, we first write

$$
\frac{\bar{q}_{\text {exp }}}{C_{\exp }}+\Phi_{0}=\frac{\Phi_{2}-\Phi_{0}+\Phi_{1}-\Phi_{0}}{2}+\Phi_{0}=\bar{\Phi}_{\exp },
$$

and note that

$$
\begin{aligned}
q_{\mathrm{imp}} & =C_{\mathrm{imp}}\left(\Phi_{\mathrm{imp}, 2}-\Phi_{2}\right)=C_{\mathrm{imp}}\left(\Phi_{\mathrm{imp}, 1}-\Phi_{1}\right) \\
& =C_{\mathrm{imp}}\left(\frac{\Phi_{\mathrm{imp}, 2}+\Phi_{\mathrm{imp}, 1}}{2}-\frac{\Phi_{2}+\Phi_{1}}{2}\right) \\
& =C_{\mathrm{imp}}\left(\bar{\Phi}_{\mathrm{imp}}-\bar{\Phi}_{\mathrm{exp}}\right) .
\end{aligned}
$$

Inserting this expression into Eq. (18), we arrive at the reaction energy as a function of potential,

$$
\Delta E=\Delta E_{0}+\left(q_{2}-q_{1}\right)\left[\bar{\Phi}_{\exp }+\frac{C_{\text {imp }}}{C_{\exp }}\left(\bar{\Phi}_{\text {imp }}-\bar{\Phi}_{\exp }\right)\right]
$$

Equation (23) demonstrates that, if the ratio $C_{\mathrm{imp}} / C$ is non-unity, the average potential 
does not uniquely describe the reaction energy. In other words, for a given potential, the reaction energy can take on multiple values depending on the charging method used to achieve that potential.

We demonstrate this finding with two examples: the Volmer reaction, and $\mathrm{CO}_{2}$ adsorption shown in Figure 6. We systematically modify the potential by varying both the explicit charge (i.e. changing the cell size and proton $/ \mathrm{CO}_{2}$ coverage) as well as with continuum charge. A third example, $\mathrm{CO}_{2}$ adsorption with varying co-adsorbates, can be found in SI Note 2. Figure 6 (a) shows that the reaction energy for Volmer is not uniquely determined by the potential, because the capacitance associated with explicit charging $\left(C_{\exp } \approx 20 \mu \mathrm{F} \mathrm{cm}^{-2}\right)$ and continuum charging $\left(C_{\mathrm{imp}} \approx 9 \mu \mathrm{F} \mathrm{cm}^{-2}\right)$ are significantly different. We note that these capacitances are fit via $q=C\left(\Phi-\Phi_{0}\right)$, as described in the first section. In contrast, Figure 6 (a) shows the reaction energy associated with $\mathrm{CO}_{2}$ adsorption as a function of potential, similarly modifying the potential both with explicit (i.e. $\mathrm{CO}_{2}$ coverage) and continuum charging. The agreement between continuum and explicit charging in this picture is much better, since the capacitance for each associated process is approximately identical $\left(C_{\mathrm{exp}} \approx 12 \mu \mathrm{F} \mathrm{cm}^{-2}, C_{\mathrm{imp}} \approx 14 \mu \mathrm{F} \mathrm{cm}^{-2}\right)$. 


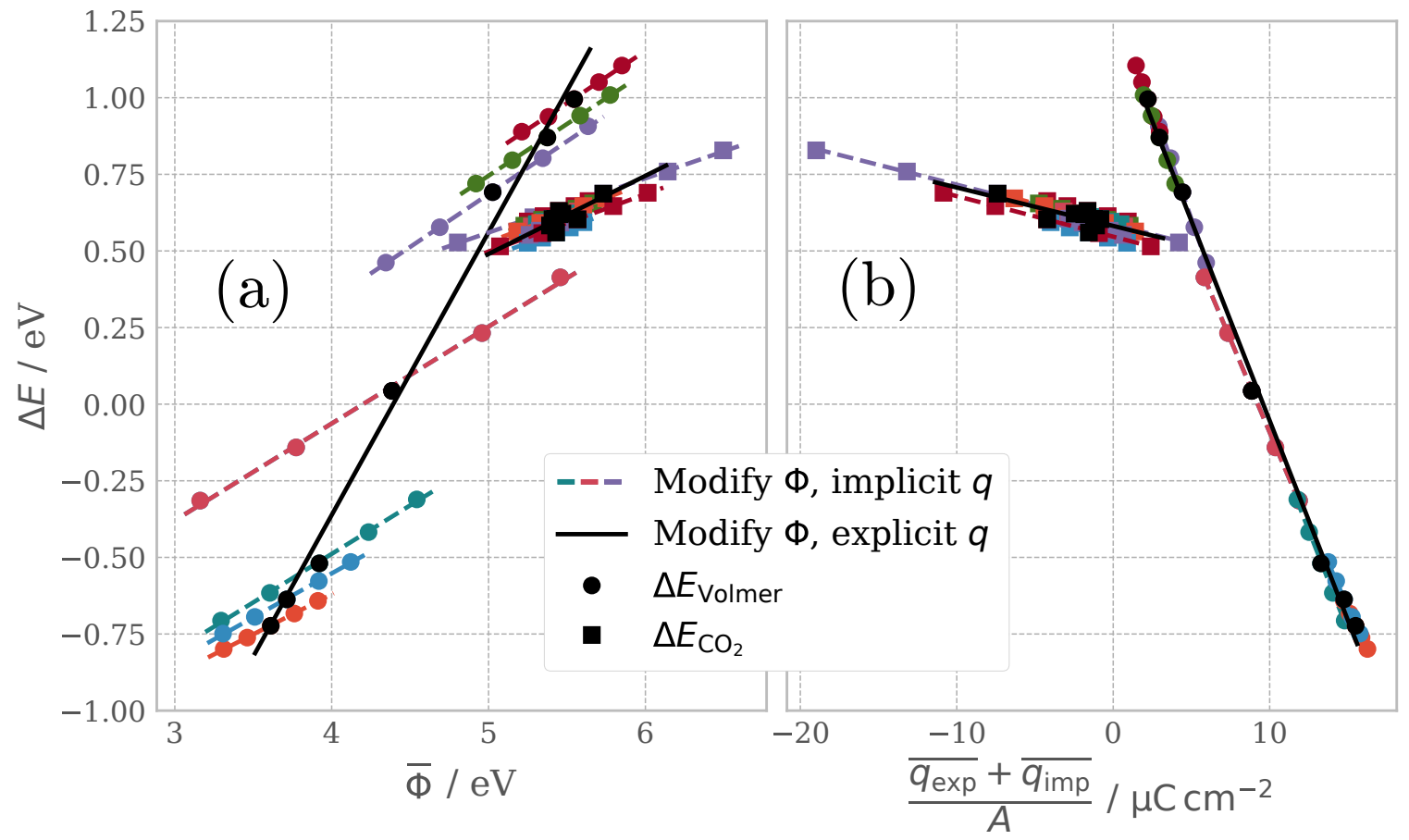

Figure 6: Reaction energetics of $\mathrm{CO}_{2}$ adsorption and the Volmer reaction as a function of (a) the average work function, corresponding to the potential as the descriptor for the driving force, as in Eq. (9). Panel (b) shows the same data set with the driving force given instead by the effective surface charge, $\frac{1}{A}\left(\bar{q}+q_{\text {imp }}\right)$, as expressed in Eq. (18). 
Figure 6 (b) then shows the energetics as a function of the effective surface charge density, corresponding to Eq. (18). Here it can be seen that regardless of the variation in capacitance, the energetics are uniquely defined. Since the capacitance for the two processes in the case of $\mathrm{CO}_{2}$ adsorption are similar, the level of noise reduction is minimal. However, for the case of the Volmer reaction, the clearly non-collinear lines collapse onto a single line. This result is because the capacitance associated with the two charging processes, i.e. explicit proton coverage and continuum countercharge concentration, are quite different $\left(C_{\exp } \approx 20 \mu \mathrm{F} \mathrm{cm}^{-2}\right.$, $C_{\mathrm{imp}} \approx 9 \mu \mathrm{F} \mathrm{cm}^{-2}$. As expressed in Eq. (23), this causes the energetics to not be uniquely described by the average work function. However, this challenge is mitigated when the same data set is instead plotted as a function of the effective surface charge. In contrast, because the capacitances associated with $\mathrm{CO}_{2}$ adsorption and the placement of continuum countercharge are quite similar, this behavior is not as clearly seen.

In principle, in the special case where the capacitance of all components are equal, then the work function again would provide single-valued functions of energy (see SI Note 3). However, tuning the parameters in the models to do so would also affect the non-electrostatic terms in the energy, such as the solvation energy. Therefore, the reparametrization of every new electrochemical process to be modeled to retain a constant capacitance would both be cumbersome and lead to inconsistent solvation parameters.

We note that although we chose subscripts exp and imp in our derivation of the model for energetics with two charging components, the model is not specific to explicit and implicit charging. An example of this model working for a case with no implicit charging is illustrated in SI Note 2, where we show the binding energy of $\mathrm{CO}_{2}$ (charging component 1) with various co-adsorbed ions (charging component 2). In this case, there is a charge associated with $\mathrm{CO}_{2}$ denoted $q_{\mathrm{CO}_{2}}$ and a charge associated with the co-adsorbate denoted $q_{\mathrm{ion}}$, which replaces subscripts exp and imp in our derivation. 


\section{Insensitivity of the Driving Force to Water Structure}

An additional challenge associated with describing the reaction energetics as a function of work function arises for the case of explicit solvation. For a given charge state, the work function of a surface can vary dramatically simply due to the varying sum of water dipoles being exerted on the surface. However, the dipole associated with a given water structure does not affect the reaction energetics. We demonstrate this in Figure 7, which shows the electric field between the surface and the first water layer for two different water structures. We calculate the field as ${ }^{40,70}$

$$
E_{\text {field }}=-\frac{d V_{\text {diff }}}{d z}
$$

where the potential difference $V_{\text {diff }}$ is defined as

$$
V_{\text {diff }}=V_{\text {total }}-V_{\text {electrolyte }}-V_{\text {slab }} .
$$

Here $V$ refers to the local electrostatic (ionic and Hartree) potential output by DFT. The subscript total refers to the local potential of the entire system, electrolyte refers to the local potential of just the electrolyte, and slab refers to the local potential of just the slab. This field reflects the local potential drop that would drive an electrochemical reaction or a chemical reaction involving polar adsorbates. In this picture, the first water layer is fixed while three water layers above have adopted different configurations that result in a nearly $4 \mathrm{eV}$ shift in the work function. Further details regarding the generation of these data can be found in Note 4 in the SI. Specifically, we demonstrate that the reaction energetics are insensitive to the change in work function associated with the changing water dipoles. 

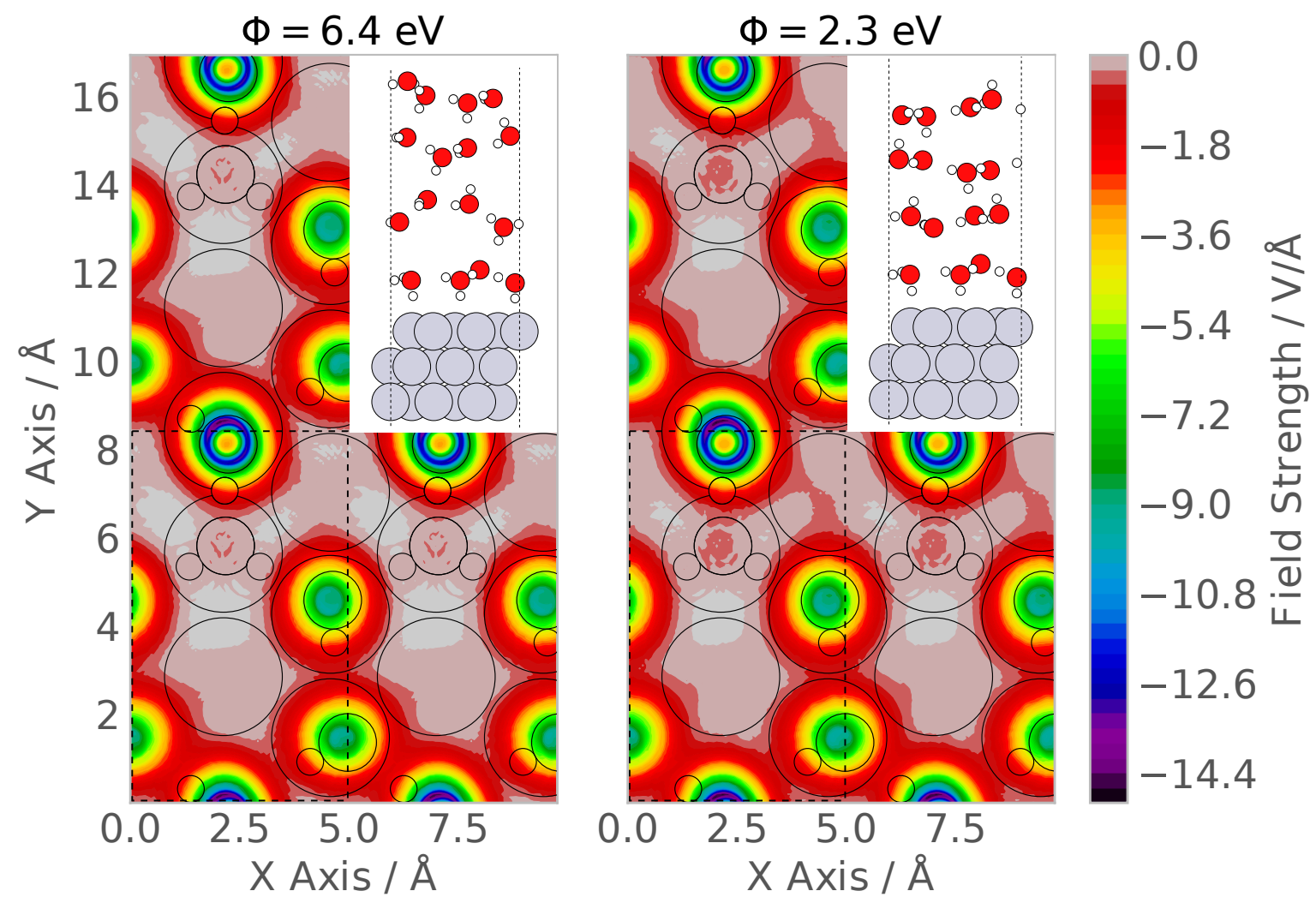

Figure 7: Electric field taken in a slice between the surface and the first water layer. Despite a nearly $4 \mathrm{eV}$ difference in the work function between the left and right panels, the electric field at the interface is virtually identical, since the surface charge is roughly the same. 


\section{Insensitivity of the Driving Force to Continuum Charge Distribu- tion}

Another consequence of the effective surface charge density being the descriptor of driving force is the insensitivity of reaction energetics to the countercharge placement. In recent years, many different models of the electrolyte distribution have emerged, which have been shown to reproduce experimental capacitance curves at varying degrees of accuracy. ${ }^{17,50-55,57-63,102}$ These charge placement models can have a significant effect on the measured capacitance ranging from 13 to $18 \mu \mathrm{F} \mathrm{cm}^{-2}$ (see SI Note 5) and potential of zero charge, and hence the predicted reaction energetics as a function of work function. However, as a function of surface charge, the energetics are largely insensitive. To demonstrate this, we have calculated the binding energy of two $\mathrm{CO}$ molecules on $\mathrm{Cu}(100)$ and $\mathrm{Cu}(211)$ using three countercharge placement models: planar countercharge, linear modified PoissonBoltzmann, and modified Poisson-Boltzmann, as implemented in Environ. ${ }^{47,103,103}$ Figure 8 illustrates that there is less than $10 \mathrm{meV}$ variation in the reaction energetics as a function of surface charge for the two facets. Note that the abscissa in $8(\mathrm{a})$ is not the effective surface charge, but just the surface charge due to continuum charging. However, because the charge due to $\mathrm{CO}$ is quite small, the effective surface charge is dominated by the continuum charging, and so we neglect to include the contribution of CO binding to the surface charge. 

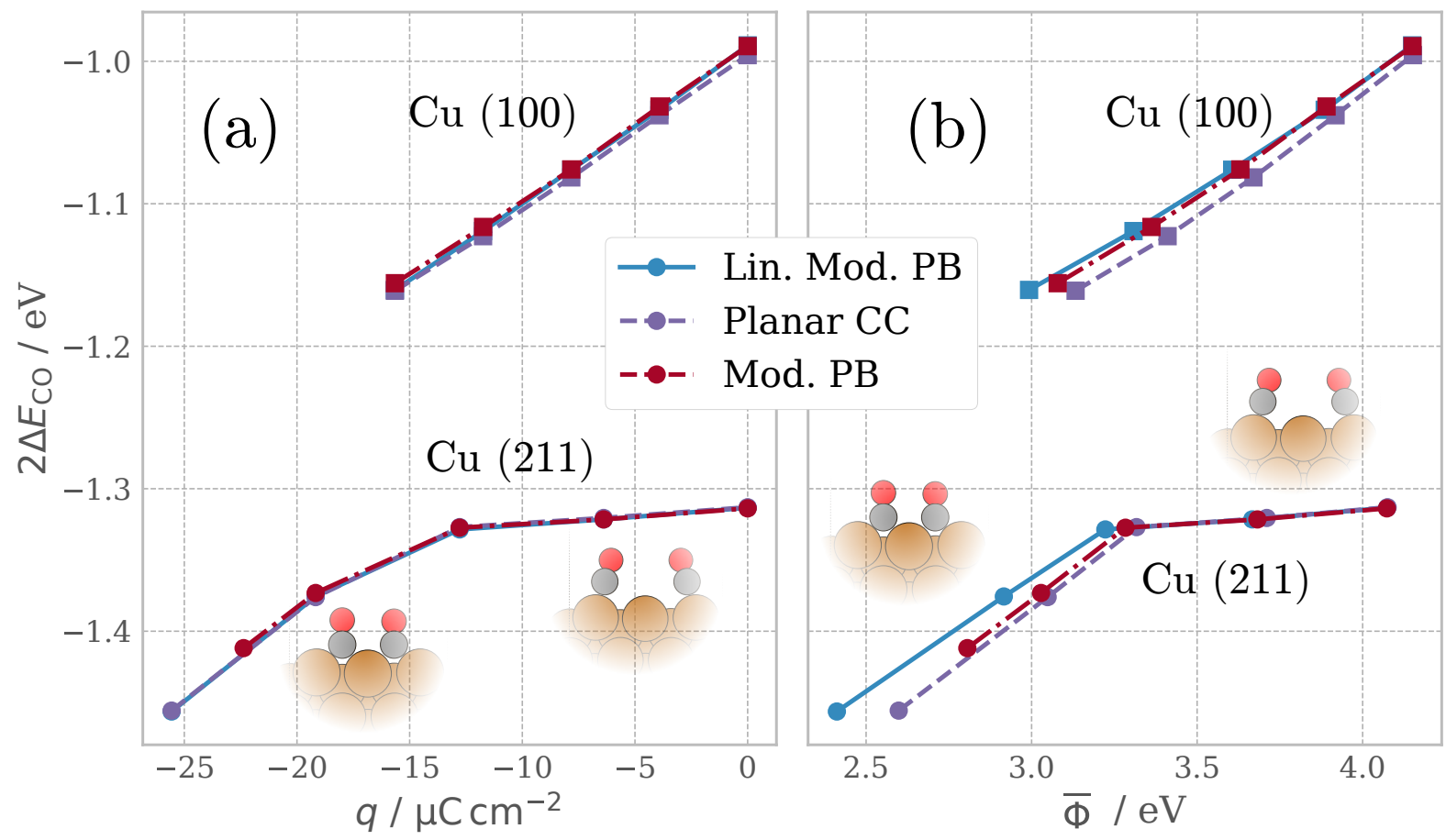

Figure 8: Illustration of the insensitivity of reaction energetics to the countercharge placement model. (a) Binding energy of two $\mathrm{CO}$ molecules on $\mathrm{Cu}$ (100) and $\mathrm{Cu}$ (211) as a function of surface charge for the three charge placement models. (b) Binding energy of two $\mathrm{CO}$ molecules on $\mathrm{Cu}(100)$ and $\mathrm{Cu}(211)$ as a function of work function. The nonlinear behavior observed for $\mathrm{Cu}(211)$ is due to a change in binding configuration as the surface charge is made more negative. 


\section{General Framework for Calculation of Electrochemical Reaction Energetics}

The models developed in this work can be applied to a general surface electrochemical reaction using a simple framework, illustrated in Figure 9.

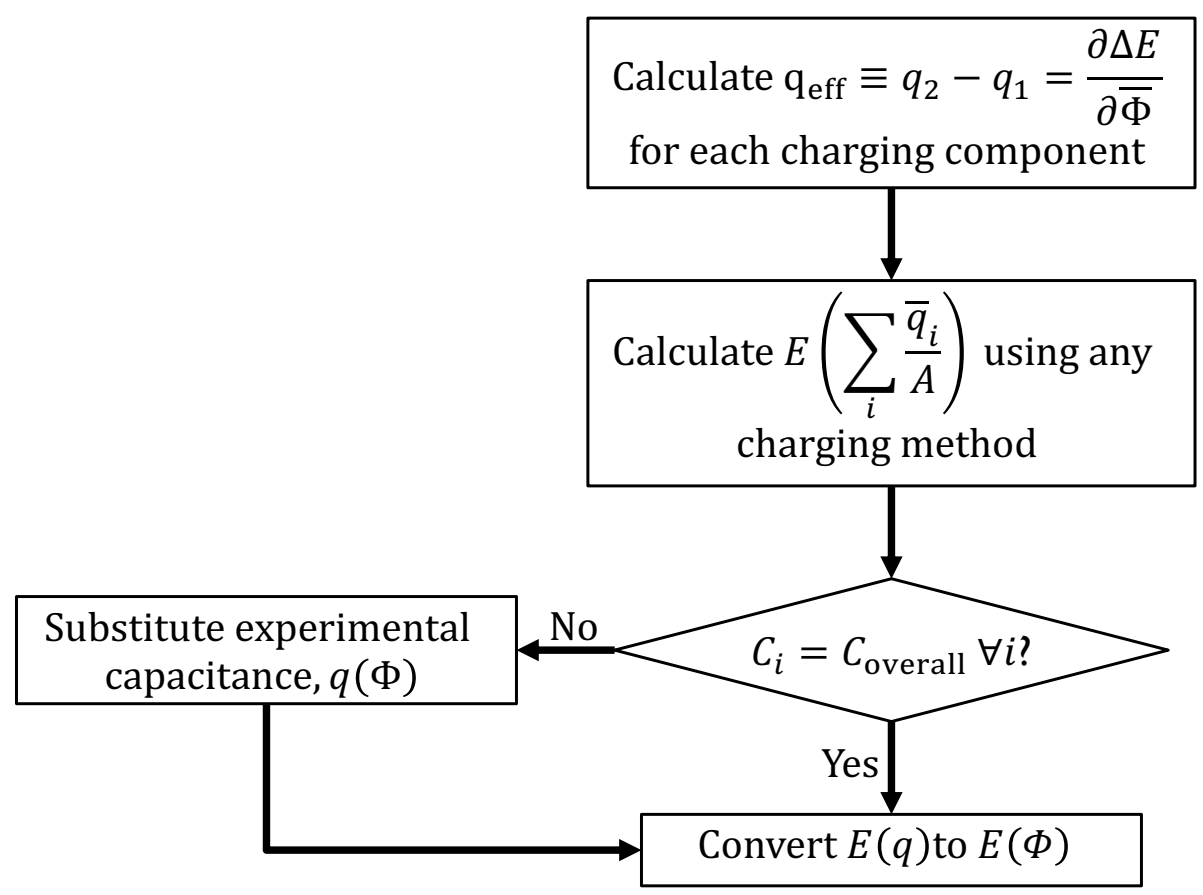

Figure 9: Flowchart illustrating the general framework for calculating electrochemical reaction energetics with the models presented in this work.

The first step, determining the effective charge $q_{\mathrm{eff}}$, is equivalent to calculating the slope of the lines shown in Figure 5. Then, determining the energetics as a function of the effective surface charge is equivalent to Figure 6 (b), where we show the Volmer reaction and $\mathrm{CO}_{2}$ adsorption as a function of the total effective surface charge. The capacitance for each charging process (in the case of Figure 6 (b), explicit and implicit charging) can be calculated by, for instant, fitting $q=C\left(\Phi-\Phi_{0}\right)$, or equivalently, fitting Eq. 13. Among the two model reactions shown, only $\mathrm{CO}_{2}$ adsorption satisfies the criteria that each capacitance is equivalent to the overall capacitance, and so the energetics as a function of charge can be directly converted to the energetics shown in Figure 6 (a). Here, the energetics are uniquely determined by the potential. However, for the second case, the Volmer reaction 
(i.e. $\left(\mathrm{H}^{+}+\mathrm{e}^{-}\right)+* \rightarrow \mathrm{H} *$ ), it cannot be assumed that the two capacitances are equivalent to the overall capacitance, since they are quite different $\left(C_{\exp } \approx 20 \mu \mathrm{F} \mathrm{cm}^{-2}, C_{\mathrm{imp}} \approx 9 \mu \mathrm{F} \mathrm{cm}^{-2}\right)$. Here, assuming an equivalent capacitance yields the energetics shown in Figure 6 (a), where it is clear that the energetics are not uniquely determined by the work function. Instead, the capacitance can be taken from an experimental charging curve for Pt (111), where it is found to have a capacitance of roughly $C \approx 20 \mu \mathrm{F} \mathrm{cm}^{-2} .{ }^{104}$ For potentials far from the potential of zero charge, a more detailed charging function $(\Phi=f(q)$ for some $f)$ could easily be substituted in lieu of the constant capacitance frequently reported in the literature (i.e. $\Phi=\frac{1}{C} q+\Phi_{0}$ ). With the energetics as a function of potential determined, they can then be input into a micro-kinetic model to determine, for instance, rates of electrochemical processes as a function of potential.

\section{Conclusions}

In this work, we have developed a general framework for the calculation of electrochemical reaction energetics from simulations of both explicit and implicit electrolyte. We demonstrate that the electrochemical interface can be effectively modeled as multiple capacitors, each corresponding to the separate charging components (i.e. separate dipole-field interactions). We show that an effective surface charge density, rather than the traditionally considered work function, is the appropriate descriptor for the driving force of electrochemical reactions. We illustrate this finding with examples of models of the Volmer reaction and $\mathrm{CO}_{2}$ adsorption. This framework allows us to, for the first time, effectively model the electrochemical interface using a hybrid explicit/continuum model in a way that is not dependent on cell size. These hybrid models are becoming increasingly popular as a way to treat the electrolyte in surface electrochemistry, since they allow for explicit treatment of charge in charge transfer reactions, without the need for sampling of solvent states. They furthermore provide a simple way to probe large ranges of potential, without the need for 
costly extrapolation techniques. Future work will apply this method to reactions occurring in basic conditions, which previously was complicated by the aforementioned band misalignment challenges ${ }^{68}$ with charged species at the interface, in addition to the usual challenges of sampling that occur with a purely explicit treatment of the electrolyte. Taken together, this work represents a significant advancement in the ability of modern computational tools to investigate surface electrochemical phenomena through the use of hybrid explicit/continuum solvation models coupled with DFT.

\section{Acknowledgement}

This material is based upon work performed by the Joint Center for Artificial Photosynthesis, a DOE Energy Innovation Hub, supported through the Office of Science of the U.S. Department of Energy under Award Number DE-SC0004993. C.F.D. acknowledges fellowship support from the National Science Foundation Graduate Research Fellowship (Grant No. DGE-114747). K.C. and H. H. H. acknowledge a research grant (9455) from "VILLUM FONDEN." Some of the computing for this project was performed on the Sherlock cluster. We would like to thank Stanford University and the Stanford Research Computing Center for providing computational resources and support that contributed to these research results. This research used resources of the National Energy Research Scientific Computing Center (NERSC), a U.S. Department of Energy Office of Science User Facility operated under Contract No. DE-AC02-05CH11231.

\section{References}

(1) Schreiner, E.; Nair, N. N.; Wittekindt, C.; Marx, D. Peptide synthesis in aqueous environments: the role of extreme conditions and pyrite mineral surfaces on formation and hydrolysis of peptides. Journal of the American Chemical Society 2011, 133 , $8216-8226$. 
(2) Rimola, A.; Sodupe, M.; Ugliengo, P. Aluminosilicate surfaces as promoters for peptide bond formation: An assessment of Bernal's hypothesis by ab Initio methods. Journal of the American Chemical Society 2007, 129, 8333-8344.

(3) Arrouvel, C.; Diawara, B.; Costa, D.; Marcus, P. DFT periodic study of the adsorption of glycine on the anhydrous and hydroxylated (0001) surfaces of $\alpha$-alumina. The Journal of Physical Chemistry C 2007, 111, 18164-18173.

(4) Irrera, S.; Costa, D.; Marcus, P. DFT periodic study of adsorption of glycine on the

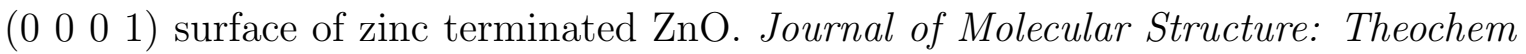
2009, 903, 49-58.

(5) Mignon, P.; Ugliengo, P.; Sodupe, M. Theoretical study of the adsorption of RNA/DNA bases on the external surfaces of Na+-montmorillonite. The Journal of Physical Chemistry C 2009, 113, 13741-13749.

(6) Jiang, D.-e.; Cooper, V. R.; Dai, S. Porous graphene as the ultimate membrane for gas separation. Nano Letters 2009, 9, 4019-4024.

(7) Hu, W.; Wu, X.; Li, Z.; Yang, J. Helium separation via porous silicene based ultimate membrane. Nanoscale 2013, 5, 9062-9066.

(8) Song, Q.; Nataraj, S.; Roussenova, M. V.; Tan, J. C.; Hughes, D. J.; Li, W.; Bourgoin, P.; Alam, M. A.; Cheetham, A. K.; Al-Muhtaseb, S. A.; Sivaniah, E. Zeolitic imidazolate framework (ZIF-8) based polymer nanocomposite membranes for gas separation. Energy \&6 Environmental Science 2012, 5, 8359-8369.

(9) Kanai, Y.; Grossman, J. C. Role of semiconducting and metallic tubes in P3HT/carbon-nanotube photovoltaic heterojunctions: density functional theory calculations. Nano letters 2008, 8, 908-912. 
(10) Appelhans, D. J.; Lin, Z.; Lusk, M. T. Two-dimensional carbon semiconductor: Density functional theory calculations. Physical Review B 2010, 82, 073410.

(11) Ludwig, T.; Gauthier, J. A.; Brown, K. S.; Ringe, S.; Norskov, J. K.; Chan, K. SolventAdsorbate Interactions and Adsorbate-Specific Solvent Structure in Carbon Dioxide Reduction on a Stepped Cu Surface. The Journal of Physical Chemistry C 2019,

(12) Heenen, H. H.; Scheurer, C.; Reuter, K.; Voss, J.; Luntz, A. C. Multi-ion Conduction in Li3OCl Glass Electrolytes. The journal of physical chemistry letters 2019,

(13) Dickens, C. F.; Nørskov, J. K. A Theoretical Investigation into the Role of Surface Defects for Oxygen Evolution on RuO2. The Journal of Physical Chemistry C 2017, 121, 18516-18524.

(14) Liu, Z.-P.; Hu, P.; Alavi, A. Catalytic role of gold in gold-based catalysts: A density functional theory study on the CO oxidation on gold. Journal of the American Chemical Society 2002, 124, 14770-14779.

(15) Yu, L.; Pan, X.; Cao, X.; Hu, P.; Bao, X. Oxygen reduction reaction mechanism on nitrogen-doped graphene: A density functional theory study. Journal of catalysis 2011, 282, 183-190.

(16) Wimmer, E.; Fu, C. L.; Freeman, A. J. Catalytic promotion and poisoning: Allelectron local-density-functional theory of CO on $\mathrm{Ni}$ (001) surfaces coadsorbed with K or S. Physical review letters 1985, 55, 2618.

(17) Mamatkulov, M.; Filhol, J.-S. An ab initio study of electrochemical vs. electromechanical properties: the case of CO adsorbed on a Pt(111) surface. Physical chemistry Chemical Physics : PCCP 2011, 13, 7675-7684.

(18) Katayama, Y.; Nattino, F.; Giordano, L.; Hwang, J.; Rao, R. R.; Andreussi, O.; Marzari, N.; Shao-Horn, Y. An In Situ Surface-Enhanced Infrared Absorption Spec- 
troscopy Study of Electrochemical CO2 Reduction: Selectivity Dependence on Surface C-Bound and O-Bound Reaction Intermediates. The Journal of Physical Chemistry C 2018, 123, 5951-5963.

(19) Heenen, H. H.; Scheurer, C.; Reuter, K. Implications of occupational disorder on ion mobility in Li4Ti5O12 battery materials. Nano letters 2017, 17, 3884-3888.

(20) Kortlever, R.; Shen, J.; Schouten, K. J. P.; Calle-Vallejo, F.; Koper, M. T. Catalysts and reaction pathways for the electrochemical reduction of carbon dioxide. The journal of physical chemistry letters 2015, 6, 4073-4082.

(21) Koper, M. T. Thermodynamic theory of multi-electron transfer reactions: Implications for electrocatalysis. Journal of Electroanalytical Chemistry 2011, 660, 254-260.

(22) Calle-Vallejo, F.; Koper, M. T. Theoretical considerations on the electroreduction of $\mathrm{CO}$ to $\mathrm{C} 2$ species on $\mathrm{Cu}(100)$ electrodes. Angewandte Chemie International Edition 2013, 52, 7282-7285.

(23) Nørskov, J. K.; Rossmeisl, J.; Logadottir, A.; Lindqvist, L.; Kitchin, J. R.; Bligaard, T.; Jonsson, H. Origin of the overpotential for oxygen reduction at a fuel-cell cathode. The Journal of Physical Chemistry B 2004, 108, 17886-17892.

(24) Liu, X.; Xiao, J.; Peng, H.; Hong, X.; Chan, K.; Nørskov, J. K. Understanding trends in electrochemical carbon dioxide reduction rates. Nature Communications 2017, 8, 15438.

(25) Tripković, V.; Skúlason, E.; Siahrostami, S.; Nørskov, J. K.; Rossmeisl, J. The oxygen reduction reaction mechanism on $\mathrm{Pt}\left(\begin{array}{llll}1 & 1 & 1\end{array}\right)$ from density functional theory calculations. Electrochimica Acta 2010, 55, 7975-7981.

(26) Nie, X.; Luo, W.; Janik, M. J.; Asthagiri, A. Reaction mechanisms of CO2 electro- 
chemical reduction on $\mathrm{Cu}\left(\begin{array}{lll}1 & 1 & 1\end{array}\right)$ determined with density functional theory. Journal of Catalysis 2014, 312, 108-122.

(27) Hyman, M. P.; Medlin, J. W. Mechanistic study of the electrochemical oxygen reduction reaction on Pt (111) using density functional theory. The Journal of Physical Chemistry B 2006, 110, 15338-15344.

(28) Vassilev, P.; Koper, M. T. Electrochemical reduction of oxygen on gold surfaces: a density functional theory study of intermediates and reaction paths. The Journal of Physical Chemistry C 2007, 111, 2607-2613.

(29) Skúlason, E.; Tripkovic, V.; Bjorketun, M. E.; Gudmundsdottir, S.; Karlberg, G.; Rossmeisl, J.; Bligaard, T.; Jónsson, H.; Nørskov, J. K. Modeling the electrochemical hydrogen oxidation and evolution reactions on the basis of density functional theory calculations. The Journal of Physical Chemistry C 2010, 114, 18182-18197.

(30) Skúlason, E.; Karlberg, G. S.; Rossmeisl, J.; Bligaard, T.; Greeley, J.; Jónsson, H.; Nørskov, J. K. Density functional theory calculations for the hydrogen evolution reaction in an electrochemical double layer on the Pt (111) electrode. Physical Chemistry Chemical Physics 2007, 9, 3241-3250.

(31) Zhang, J.; Wang, Z.; Zhu, Z. The inherent kinetic electrochemical reduction of oxygen into H2O on FeN4-carbon: a density functional theory study. Journal of Power Sources 2014, 255, 65-69.

(32) Yeh, K.-Y.; Janik, M. J. Density functional theory-based electrochemical models for the oxygen reduction reaction: Comparison of modeling approaches for electric field and solvent effects. Journal of computational chemistry 2011, 32, 3399-3408.

(33) Shi, C.; Chan, K.; Yoo, J. S.; Nørskov, J. K. Barriers of electrochemical CO2 reduction on transition metals. Organic Process Research $\& 3$ Development 2016, 20, 1424-1430. 
(34) Lessio, M.; Senftle, T. P.; Carter, E. A. Hydride Shuttle Formation and Reaction with CO2 on GaP (110). ChemSusChem 2018, 11, 1558-1566.

(35) Liao, P.; Keith, J. A.; Carter, E. A. Water oxidation on pure and doped hematite (0001) surfaces: Prediction of $\mathrm{Co}$ and $\mathrm{Ni}$ as effective dopants for electrocatalysis. Journal of the American Chemical Society 2012, 134, 13296-13309.

(36) Sakong, S.; Groß, A. The importance of the electrochemical environment in the electrooxidation of methanol on Pt (111). ACS Catalysis 2016, 6, 5575-5586.

(37) Stecher, T.; Reuter, K.; Oberhofer, H. First-Principles Free-Energy Barriers for Photoelectrochemical Surface Reactions: Proton Abstraction at TiO 2 (110). Physical review letters 2016, 117, 276001.

(38) Montoya, J. H.; Shi, C.; Chan, K.; Nørskov, J. K. Theoretical insights into a CO dimerization mechanism in CO2 electroreduction. The Journal of Physical Chemistry Letters 2015, 6, 2032-2037.

(39) Karlberg, G.; Rossmeisl, J.; Nørskov, J. K. Estimations of electric field effects on the oxygen reduction reaction based on the density functional theory. Physical Chemistry Chemical Physics 2007, 9, 5158-5161.

(40) Chen, L. D.; Urushihara, M.; Chan, K.; Nørskov, J. K. Electric field effects in electrochemical CO2 reduction. ACS Catalysis 2016, 6, 7133-7139.

(41) Strmcnik, D.; Uchimura, M.; Wang, C.; Subbaraman, R.; Danilovic, N.; Van Der Vliet, D.; Paulikas, A. P.; Stamenkovic, V. R.; Markovic, N. M. Improving the hydrogen oxidation reaction rate by promotion of hydroxyl adsorption. Nature chemistry 2013, 5, 300 .

(42) Liu, X.; Schlexer, P.; Xiao, J.; Ji, Y.; Wang, L.; Sandberg, R. B.; Tang, M.; Brown, K. S.; Peng, H.; Ringe, S.; Hahn, C.; Jaramillo, T. F.; Nørskov, J. K.; Chan, K. 
$\mathrm{pH}$ effects on the electrochemical reduction of $\mathrm{CO}(2)$ towards $\mathrm{C} 2$ products on stepped copper. Nature Communications 2019, 10, 32.

(43) Rossmeisl, J.; Chan, K.; Ahmed, R.; Tripković, V.; Björketun, M. E. pH in atomic scale simulations of electrochemical interfaces. Physical Chemistry Chemical Physics 2013, 15, 10321-10325.

(44) Rossmeisl, J.; Skúlason, E.; Björketun, M. E.; Tripkovic, V.; Nørskov, J. K. Modeling the electrified solid-liquid interface. Chemical Physics Letters 2008, 466, 68-71.

(45) Chan, K.; Nørskov, J. K. Potential dependence of electrochemical barriers from ab initio calculations. The Journal of Physical Chemistry Letters 2016, 7, 1686-1690.

(46) Chan, K.; Nørskov, J. K. Electrochemical barriers made simple. The Journal of Physical Chemistry Letters 2015, 6, 2663-2668.

(47) Andreussi, O.; Dabo, I.; Marzari, N. Revised self-consistent continuum solvation in electronic-structure calculations. The Journal of Chemical Physics 2012, 136, 064102.

(48) Kastlunger, G.; Lindgren, P.; Peterson, A. A. Controlled-potential simulation of elementary electrochemical reactions: Proton discharge on metal surfaces. The Journal of Physical Chemistry C 2018, 122, 12771-12781.

(49) Otani, M.; Sugino, O. First-principles calculations of charged surfaces and interfaces: A plane-wave nonrepeated slab approach. Physical Review B 2006, 73, 115407.

(50) Lozovoi, A.; Alavi, A.; Kohanoff, J.; Lynden-Bell, R. Ab initio simulation of charged slabs at constant chemical potential. The Journal of Chemical Physics 2001, 115, 1661-1669.

(51) Taylor, C. D.; Wasileski, S. A.; Filhol, J.-S.; Neurock, M. First principles reaction modeling of the electrochemical interface: Consideration and calculation of a tunable 
surface potential from atomic and electronic structure. Physical Review B 2006, 73, 165402.

(52) Fang, Y.-H.; Liu, Z.-P. Surface phase diagram and oxygen coupling kinetics on flat and stepped Pt surfaces under electrochemical potentials. The Journal of Physical Chemistry C 2009, 113, 9765-9772.

(53) Fang, Y.-H.; Liu, Z.-P. Mechanism and tafel lines of electro-oxidation of water to oxygen on RuO2 (110). Journal of the American Chemical Society 2010, 132, 1821418222.

(54) Ringe, S.; Oberhofer, H.; Hille, C.; Matera, S.; Reuter, K. Function-space-based solution scheme for the size-modified Poisson-Boltzmann equation in full-potential DFT. Journal of Chemical Theory and Computation 2016, 12, 4052-4066.

(55) Jinnouchi, R.; Anderson, A. B. Aqueous and surface redox potentials from selfconsistently determined gibbs energies. Journal of Physical Chemistry C 2008, 112, $8747-8750$.

(56) Mathew, K.; Sundararaman, R.; Letchworth-Weaver, K.; Arias, T.; Hennig, R. G. Implicit solvation model for density-functional study of nanocrystal surfaces and reaction pathways. The Journal of Chemical Physics 2014, $140,084106$.

(57) Mathew, K.; Hennig, R. G. Implicit self-consistent description of electrolyte in planewave density-functional theory. arXiv preprint arXiv:1601.03346 2016,

(58) Fisicaro, G.; Genovese, L.; Andreussi, O.; Marzari, N.; Goedecker, S. A generalized Poisson and Poisson-Boltzmann solver for electrostatic environments. The Journal of Chemical Physics 2016, 144, 014103.

(59) Fang, Y.-H.; Wei, G.-F.; Liu, Z.-P. Theoretical modeling of electrode/electrolyte inter- 
face from first-principles periodic continuum solvation method. Catalysis Today 2013, 202, 98-104.

(60) Letchworth-Weaver, K.; Arias, T. Joint density functional theory of the electrodeelectrolyte interface: Application to fixed electrode potentials, interfacial capacitances, and potentials of zero charge. Physical Review B 2012, 86, 075140.

(61) Keilbart, N.; Okada, Y.; Feehan, A.; Higai, S.; Dabo, I. Quantum-continuum simulation of the electrochemical response of pseudocapacitor electrodes under realistic conditions. Physical Review B 2017, 95, 115423.

(62) Sundararaman, R.; Schwarz, K. Evaluating continuum solvation models for the electrode-electrolyte interface: Challenges and strategies for improvement. The Journal of Chemical Physics 2017, 146, 084111.

(63) Minezawa, N.; Kato, S. Efficient implementation of three-dimensional reference interaction site model self-consistent-field method: Application to solvatochromic shift calculations. The Journal of Chemical Physics 2007, 126, 054511.

(64) Nattino, F.; Truscott, M.; Marzari, N.; Andreussi, O. Continuum models of the electrochemical diffuse layer in electronic-structure calculations. The Journal of chemical physics 2019, 150, 041722.

(65) Gauthier, J.; Dickens, C. F.; Chen, L. D.; Doyle, A. D.; Norskov, J. K. Solvation Effects for Oxygen Evolution Reaction Catalysis on $\mathrm{IrO}_{2}$ (110). Journal of Physical Chemistry C 2017, 2.

(66) Kristoffersen, H. H.; Vegge, T.; Hansen, H. A. OH formation and H 2 adsorption at the liquid water-Pt (111) interface. Chemical Science 2018, 9, 6912-6921.

(67) Sakong, S.; Groß, A. The electric double layer at metal-water interfaces revisited based on a charge polarization scheme. The Journal of Chemical Physics 2018, 149, 084705. 
(68) Björketun, M. E.; Zeng, Z.; Ahmed, R.; Tripkovic, V.; Thygesen, K. S.; Rossmeisl, J. Avoiding pitfalls in the modeling of electrochemical interfaces. Chemical Physics Letters 2013, 555, 145-148.

(69) Perdew, J. P. Density functional theory and the band gap problem. International Journal of Quantum Chemistry 1985, 28, 497-523.

(70) Gauthier, J. A.; Ringe, S.; Dickens, C. F.; Garza, A. J.; Bell, A. T.; Head-Gordon, M.; Nørskov, J. K.; Chan, K. Challenges in Modeling Electrochemical Reaction Energetics with Polarizable Continuum Models. ACS Catalysis 2018, 9, 920-931.

(71) Ringe, S.; Oberhofer, H.; Reuter, K. Transferable ionic parameters for first-principles Poisson-Boltzmann solvation calculations: Neutral solutes in aqueous monovalent salt solutions. The Journal of Chemical Physics 2017, 146, 134103.

(72) Karlberg, G. Adsorption trends for water, hydroxyl, oxygen, and hydrogen on transition-metal and platinum-skin surfaces. Physical Review B 2006, 74, 153414.

(73) Rossmeisl, J.; Greeley, J.; Karlberg, G. Electrocatalysis and catalyst screening from density functional theory calculations. Fuel Cell Catalysis: A Surface Science Approach 2009, 57-92.

(74) Briquet, A. L.; Sarwar, M.; Mugo, J.; Jones, G.; Calle-vallejo, F.; Briquet, L. G. V.; Sarwar, M.; Mugo, J.; Jones, G.; Calle-vallejo, F. A new type of scaling relations to assess the accuracy of computational predictions of catalytic activities applied to the oxygen evolution reaction.

(75) Goodpaster, J. D.; Bell, A. T.; Head-Gordon, M. Identification of possible pathways for $\mathrm{C}-\mathrm{C}$ bond formation during electrochemical reduction of $\mathrm{CO} 2$ : New theoretical insights from an improved electrochemical model. The Journal of Physical Chemistry Letters 2016, 7, 1471-1477. 
(76) Saleheen, M.; Heyden, A. Liquid Phase Modeling in Heterogeneous Catalysis. ACS Catalysis 2018,

(77) Jensen, F. Introduction of Computational Chemistry, 2nd ed.; John Wiley \& Sons: Chichester, 2007.

(78) Tang, W.; Sanville, E.; Henkelman, G. A grid-based Bader analysis algorithm without lattice bias. Journal of Physics: Condensed Matter 2009, 21, 084204.

(79) Sanville, E.; Kenny, S. D.; Smith, R.; Henkelman, G. Improved grid-based algorithm for Bader charge allocation. Journal of Computational Chemistry 2007, 28, 899-908.

(80) Henkelman, G.; Arnaldsson, A.; Jónsson, H. A fast and robust algorithm for Bader decomposition of charge density. Computational Materials Science 2006, 36, 354-360.

(81) Frumkin, A. Wasserstoffüberspannung und Struktur der Doppelschicht. Zeitschrift für physikalische Chemie 1933, 164, 121-133.

(82) Van Soestbergen, M. Frumkin-Butler-Volmer theory and mass transfer in electrochemical cells. Russian journal of electrochemistry 2012, 48, 570-579.

(83) Kresse, G.; Hafner, J. Ab initio molecular dynamics for liquid metals. Physical Review $B$ 1993, 47, 558-561.

(84) Kresse, G.; Furthmüller, J. Efficient iterative schemes for ab initio total-energy calculations using a plane-wave basis set. Physical Review B 1996, 54, 11169-11186.

(85) Kresse, G.; Furthmüller, J. Efficiency of ab-initio total energy calculations for metals and semiconductors using a plane-wave basis set. Computational Materials Science 1996, $6,15-50$.

(86) Giannozzi, P. et al. QUANTUM ESPRESSO: A Modular and Open-Source Software Project for Quantum Simulations of Materials. Journal of Physics: Condensed Matter 2009, 21, 395502. 
(87) Garrity, K. F.; Bennett, J. W.; Rabe, K. M.; Vanderbilt, D. Pseudopotentials for highthroughput DFT calculations. Computational Materials Science 2014, 81, 446-452.

(88) Hammer, B.; Hansen, L. B.; Nørskov, J. K. Improved adsorption energetics within density-functional theory using revised Perdew-Burke-Ernzerhof functionals. Physical Review B 1999, 59, 7413.

(89) Monkhorst, H. J.; Pack, J. D. Special points for Brillouin-zone integrations. Physical Review B 1976, 13, 5188.

(90) Gauthier, J.; Dickens, C.; Ringe, S.; Chan, K. Practical Considerations for Continuum Models Applied to Surface Electrochemistry. 2019,

(91) Chen, L. D.; Bajdich, M.; Martirez, J. M. P.; Krauter, C. M.; Gauthier, J. A.; Carter, E. A.; Luntz, A. C.; Chan, K.; Nørskov, J. K. Understanding the apparent fractional charge of protons in the aqueous electrochemical double layer. Nature Communications 2018, 9, 3202 .

(92) de Levie, R. The electrosorption valency and partial charge transfer. Journal of Electroanalytical Chemistry 2004, 562, 273-276.

(93) Grahame, D. C. Components of charge and potential in the non-diffuse region of the electrical double layer: potassium iodide solutions in contact with mercury at 25 . Journal of the American Chemical Society 1958, 80, 4201-4210.

(94) Schultze, J.; Rolle, D. The partial discharge of electrosorbates and its influence in electrocatalysis. Canadian journal of chemistry 1997, 75, 1750-1758.

(95) Dabo, I.; Li, Y.; Bonnet, N.; Marzari, N. Ab initio electrochemical properties of electrode surfaces. Fuel Cell Science: Theory, Fundamentals, and Biocatalysis 2010, 415431. 
(96) Bonnet, N.; Dabo, I.; Marzari, N. Chemisorbed molecules under potential bias: detailed insights from first-principles vibrational spectroscopies. Electrochimica Acta 2014, 121, 210-214.

(97) Filhol, J.-S.; Doublet, M.-L. An ab initio study of surface electrochemical disproportionation: The case of a water monolayer adsorbed on a Pd (llll 111$)$ surface. Catalysis Today 2013, 202, 87-97.

(98) Steinmann, S. N.; Michel, C.; Schwiedernoch, R.; Sautet, P. Impacts of electrode potentials and solvents on the electroreduction of CO 2: a comparison of theoretical approaches. Physical Chemistry Chemical Physics 2015, 17, 13949-13963.

(99) Van den Bossche, M.; Skúlason, E.; Rose-Petruck, C.; Jónsson, H. Assessment of Constant-Potential Implicit Solvation Calculations of Electrochemical Energy Barriers for H2 Evolution on Pt. The Journal of Physical Chemistry C 2019,

(100) Steinmann, S. N.; Sautet, P. Assessing a first-principles model of an electrochemical interface by comparison with experiment. The Journal of Physical Chemistry C 2016, 120, 5619-5623.

(101) Sundararaman, R.; Letchworth-Weaver, K.; Schwarz, K. A.; Gunceler, D.; Ozhabes, Y.; Arias, T. JDFTx: software for joint density-functional theory. SoftwareX 2017, 6, 278-284.

(102) Borukhov, I.; Andelman, D.; Orland, H. Adsorption of large ions from an electrolyte solution: a modified Poisson-Boltzmann equation. Electrochimica Acta 2000, 46, 221229.

(103) Andreussi, O.; Hormann, N. G.; Nattino, F.; Fisicaro, G.; Goedecker, S.; Marzari, N. Solvent-aware interfaces in continuum solvation. Journal of chemical theory and computation 2019, 
(104) Pajkossy, T.; Kolb, D. M. Double layer capacitance of Pt ( 111 ) single crystal electrodes. Electrochimica Acta 2001, 46, 3063-3071. 
Graphical TOC Entry

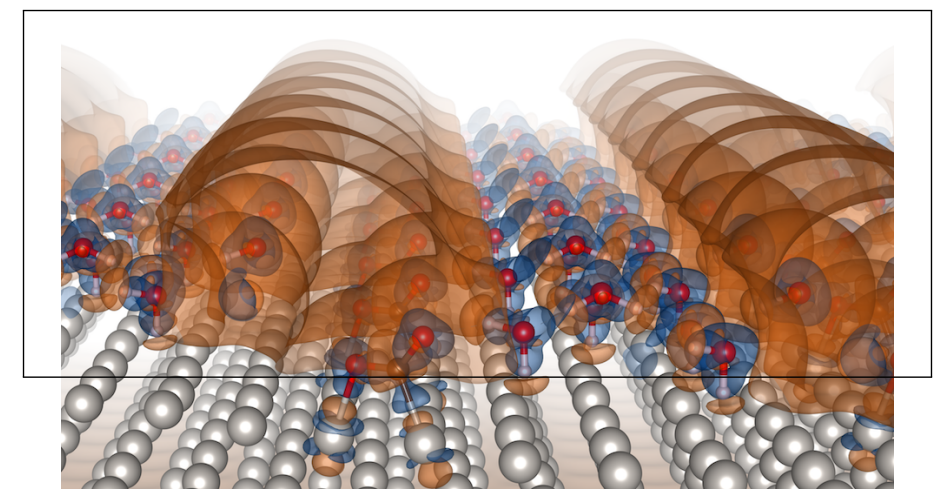




\section{Supporting Information: Unified approach to}

implicit and explicit solvent simulations of electrochemical reaction energetics

Joseph A. Gauthier, ${ }^{\dagger, \downarrow}$ Colin F. Dickens, ${ }^{\dagger, \ddagger}$ Hendrik H. Heenen, "Stefan Ringe,,,$+ \dagger$ and Karen Chan ${ }^{*, \Phi}$

$\dagger$ SUNCAT Center for Interface Science and Catalysis, Department of Chemical

Engineering, Stanford University, Stanford, California 94305, United States

$\ddagger S U N C A T$ Center for Interface Science and Catalysis, SLAC National Accelerator

Laboratory, 2575 Sand Hill Road, Menlo Park, California 94025, United States

\Department of Physics, Technical University of Denmark, DK-2800, Kgs. Lyngby, Denmark

E-mail: kchan@fysik.dtu.dk 


\section{Note 1}

In this note, we further explain the use of the average work function, $\bar{\Phi}$, as the descriptor for electrochemical reactions used in the main text.

In the main text, we establish that the energy depends quadratically on the surface charge density $q$ and the potential $\Phi$. That is, we assume that the capacitance is a constant function of potential and therefore the cubic term in the Taylor expansion is negligible (this is shown to be a good assumption in Figure 4). Expanding the energy as a function of $q$, we have

$$
E(q)=E(q=0)+q \Phi_{0}+\frac{q^{2}}{2 C}
$$

We can write this instead as a function of potential by using the usual capacitance relation $q=C\left(\Phi-\Phi_{0}\right)$

$$
E(\Phi)=E\left(\Phi=\Phi_{0}\right)+C \Phi_{0}\left(\Phi-\Phi_{0}\right)+\frac{C}{2}\left(\Phi-\Phi_{0}\right)^{2}
$$

Seen in Figure S1 is a plot of the energy $E$ as a function of the coverage $\theta$ of (in this case) protons. In general, the energy depends quadratically on both $\theta$ and work function $\Phi .^{1}$ In practical density functional theory (DFT) calculations of relative energies between two different states of $\theta$ or $\Phi$, the change in energy calculated is not equal to $d E / d \theta$. Instead it is equal to $\Delta E / \Delta \theta$, since the coverage changes substantially between the initial and final state. This is illustrated in Figure S1, where the blue dashed line indicates the energy change between two DFT calculations with different coverages (theta=0 and 0.5 ). What one would like to calculate though is instead the instantaneous derivative, in order to relate energy differences to a coverage or work function. Toward this end, we take advantage of a property of parabolas, for which the slope of a secant line is equivalent to the slope of the tangent at the average value of the independent variable. For a general parabola $f(x)=c_{1} x^{2}+c_{2} x+c_{3}$, 
the slope of a secant line from point $a$ to $b$ is given by

$$
\begin{aligned}
\frac{\Delta f(x)}{\Delta x} & =\frac{f(b)-f(a)}{b-a} \\
& =\frac{c_{1}\left(b^{2}-a^{2}\right)+c_{2}(b-a)}{b-a} \\
& =c_{1}(b+a)+c_{2}
\end{aligned}
$$

The slope of the tangent line at a given point $x$ is then given by

$$
\frac{d f}{d x}=2 c_{1} x+c_{2}
$$

Equating (S3) and (S4), we see that the slope of the tangent line and secant line are equivalent for the tangent to the average of $a$ and $b$, i.e.

$$
\frac{d f}{d x}\left(x=\frac{a+b}{2}\right)=\frac{f(b)-f(a)}{b-a}
$$




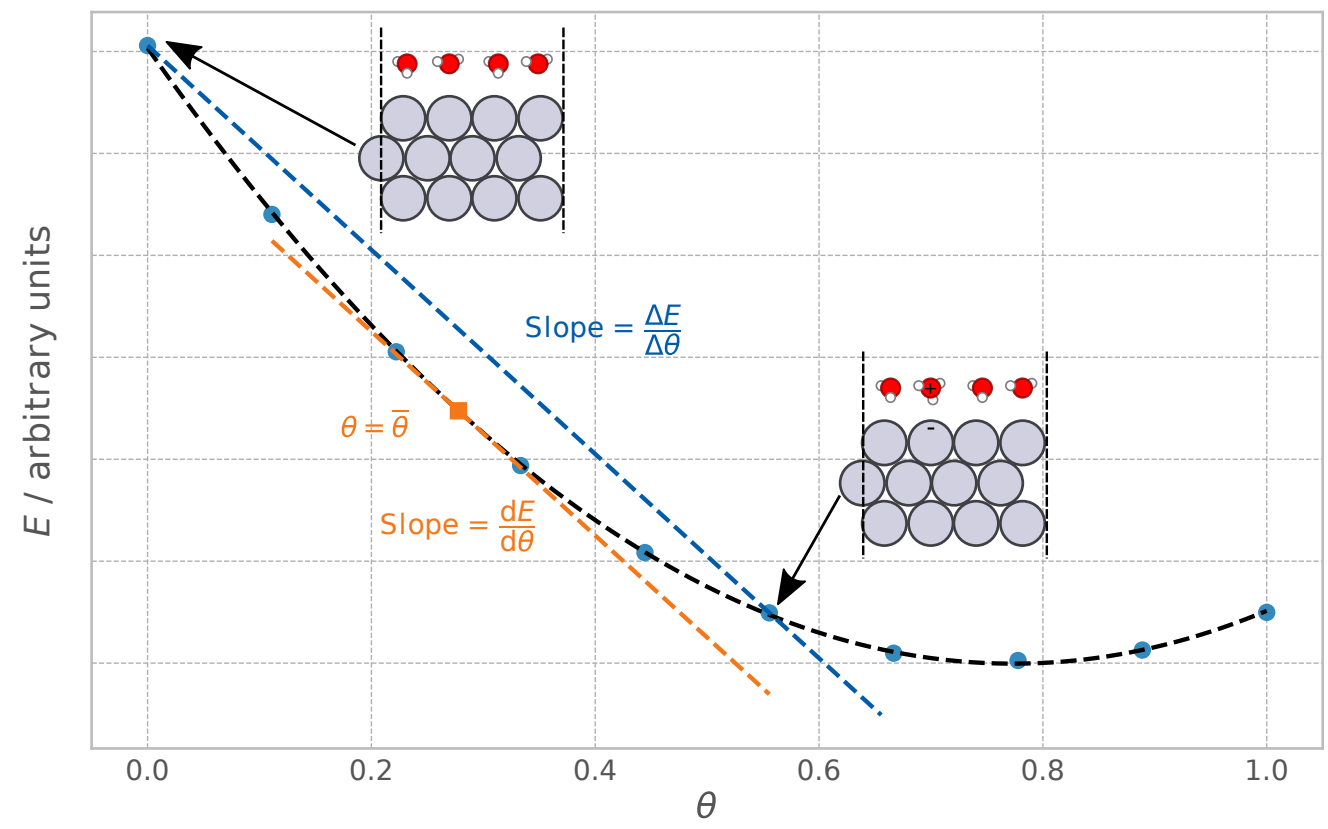

Figure S1: Graphic explanation of the average work function being used to describe reaction energetics. In this graphic, the coverage of protons $\theta$ is used to illustrate the concept. Since the energy $E$ depends quadratically on $\theta$, the slope of the secant line between two points $\theta_{1}$ and $\theta_{2}$ is exactly equal to the slope of the tangent line at $\theta=\bar{\theta}$.

In the context of Figure S1, this means that the change in energy calculated between two states in a standard DFT calculation (in the Figure, going from $\theta=0$ to $\theta=0.5$ ) is equivalent to the DFT calculation with no change in coverage between initial and final states (i.e. cell-extrapolated), at a coverage of $\theta=\bar{\theta}=0.25$.

Because the energy also depends quadratically on the work function $\Phi$, the same argument is made. The change in energy as calculated by DFT in a finite cell, which results in a change in work function $\Phi_{1} \rightarrow \Phi_{2}$, is exactly equivalent to the calculation with no change in work function, at the average work function. By analogy to Eq. (S5),

$$
\begin{gathered}
\frac{d E}{d \theta}(\theta=\bar{\theta})=\frac{E\left(\theta_{2}\right)-E\left(\theta_{1}\right)}{\theta_{2}-\theta_{1}} \\
\frac{d E}{d \Phi}(\Phi=\bar{\Phi})=\frac{E\left(\Phi_{2}\right)-E\left(\Phi_{1}\right)}{\Phi_{2}-\Phi_{1}} .
\end{gathered}
$$




\section{Note 2}

Here we illustrate a third example of surface charge as the descriptor for the driving force of charge transfer processes. Shown in Figure $\mathrm{S} 2$ is the binding energy of two $\mathrm{CO}_{2}$ molecules as both a function of the average work function, and as a function of the average effective charge. Note that the binding energy for two $\mathrm{CO}_{2}$ molecules is shown because a cell symmetric in the $z$-coordinate was used.
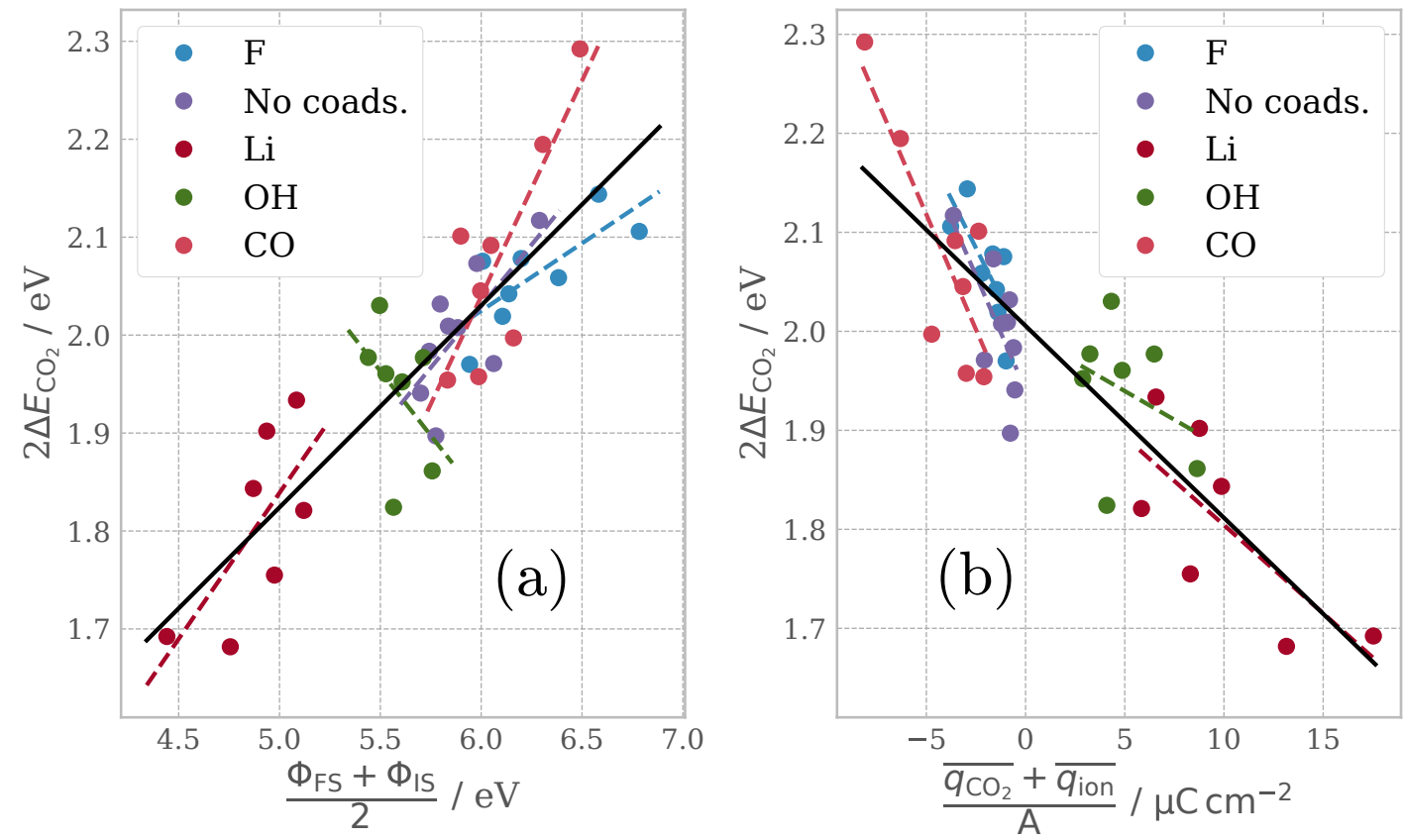

Figure S2: Binding energy of $\mathrm{CO}_{2}$ on $\mathrm{Pt}$ (111) with various coadsorbates to modify the work function. (a) $\Delta E_{\mathrm{CO}_{2}}$ as a function of the average work function. (b) $\Delta E_{\mathrm{CO}_{2}}$ as a function of the effective surface charge. The solid black line represents a best fit to all data points.

As can be seen in Figure S2(a), the data shows a significant amount of noise. This is due largely to the chemical interaction between the coadsorbate and $\mathrm{CO}_{2}$ on the surface. We note that the scale on the y-axis is rather small, and so the average deviation remains quite small overall. Figure S2(b) shows some reduced noise, with all slopes having the same sign. As outlined in the main text, the effective charge $q_{\mathrm{eff}}$, due to the ion $\left(\right.$ and $\left.\mathrm{CO}_{2}\right)$ is determined via

$$
q_{\mathrm{eff}}=\frac{\partial \Delta E_{\text {ion }}}{\partial \bar{\Phi}} .
$$




\section{Note 3}

In this note, we discuss tuning the capacitance of the implicit model, such that it matches that of the overall process. In principle this would mitigate the issues presented in the main text, where the work function acts as a poor descriptor for the driving force. If the capacitance for every charging process in the system is the same, then the work function can act as a descriptor. We found that using a partial bilayer of explicit solvent (rather than a full bilayer), equivalent to only one water molecule to stabilize each hydronium ion, lowered the capacitance due to explicit protons.
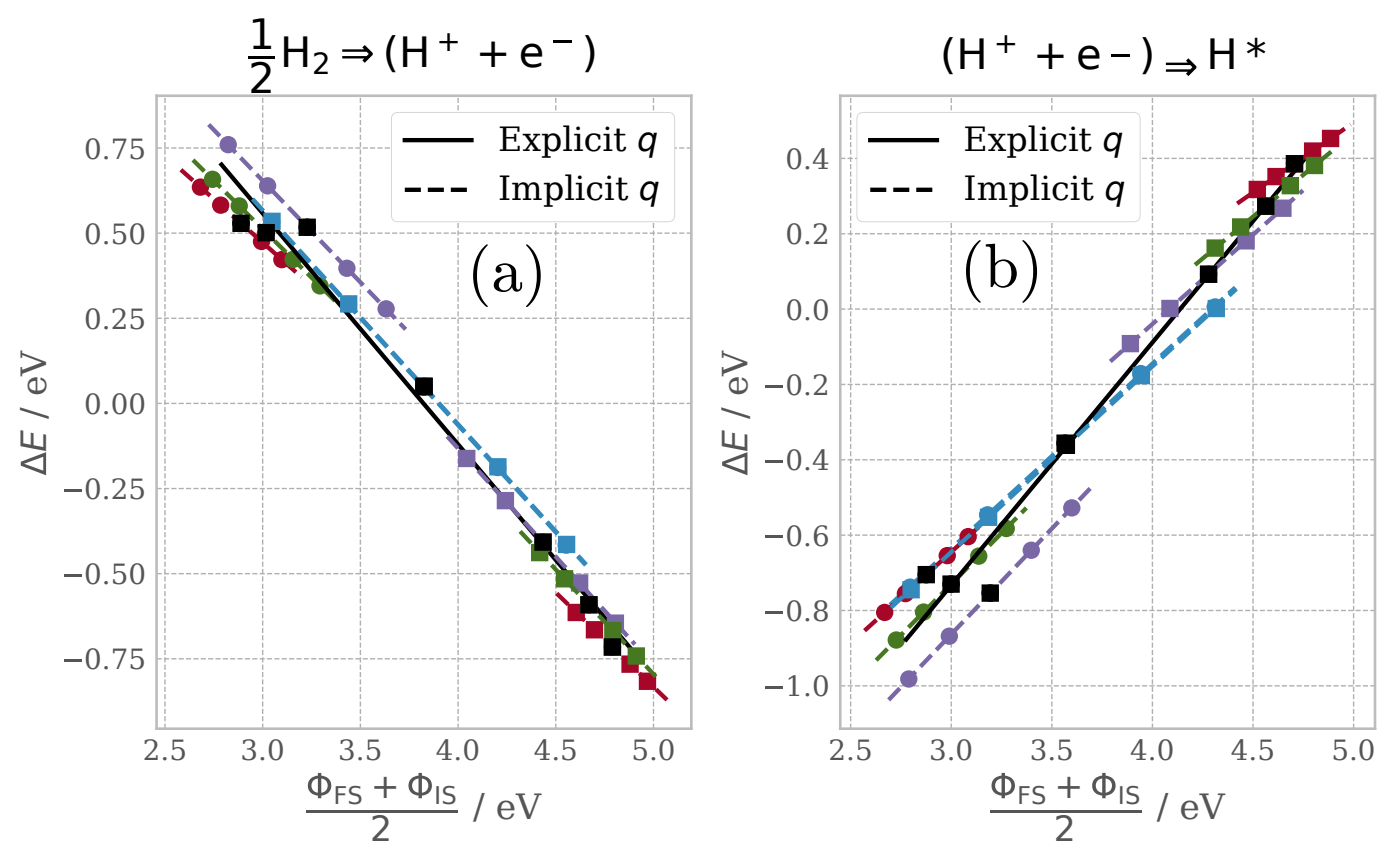

Figure S3: Illustration of capacitance tuning for (a) proton creation and (b) the Volmer reaction. Here the dashed colored lines correspond to different fixed proton concentrations, with the work function being modified by changing the continuum countercharge. The dashed line corresponds to the case of zero continuum countercharge, with the work function being modified by changing the explicit proton coverage (i.e. cell extrapolation).

As can be seen in Figure S3, using a very sparse bilayer sufficiently lowers the capacitance of explicit charging, to the point that it roughly matches that of implicit charging. This results in the work function acting as a better descriptor for the driving force, as evidenced by the lines for implicit and explicit charging being approximately parallel and having similar 
intercepts. Figure S4 illustrates the problem with this approach of tuning the capacitance. Here we plot the Volmer reaction energy as a function of (a) the proton coverage $\theta$ and (b) work function $\Phi$.
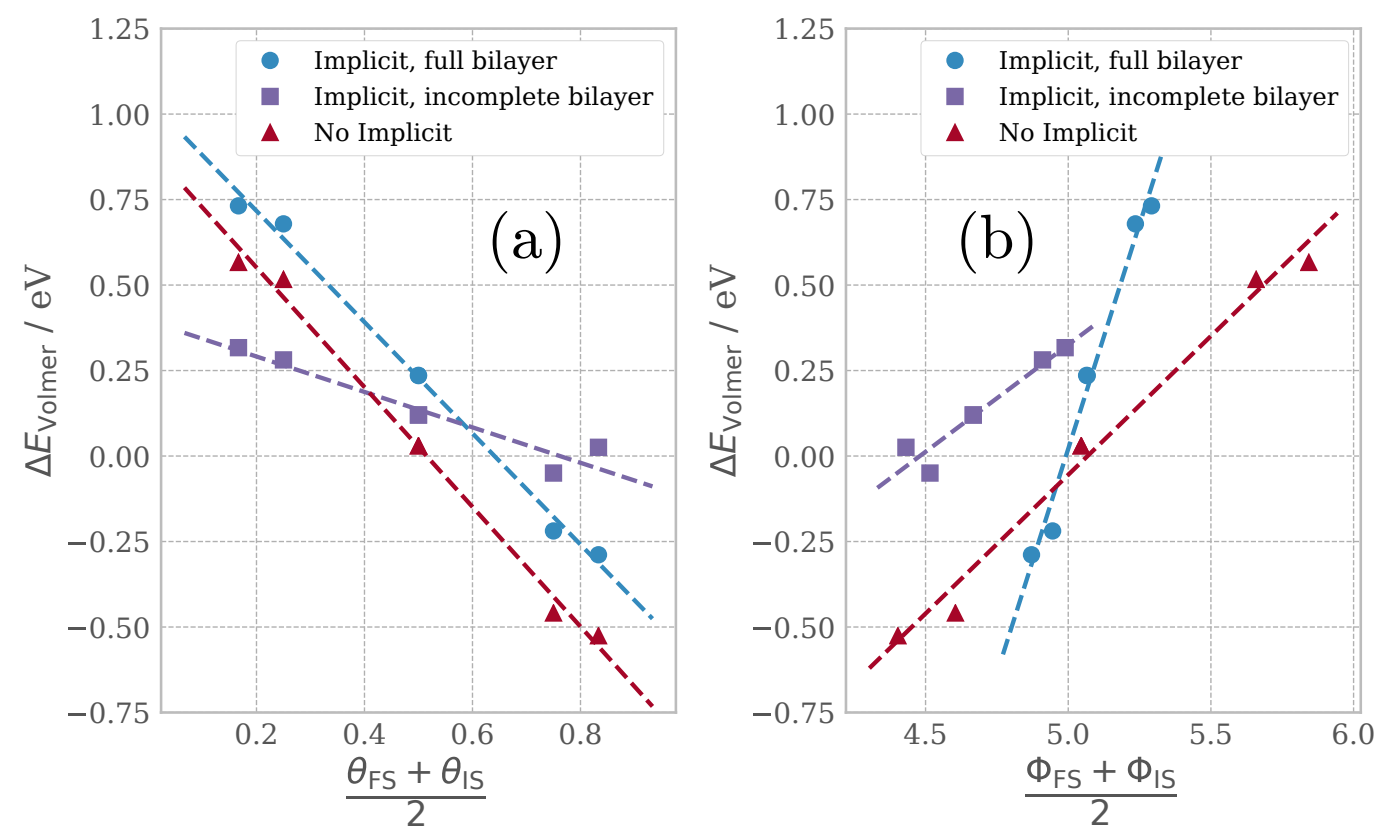

Figure S4: Illustration of the problem using an incomplete bilayer to tune the capacitance. (a) Change in Volmer reaction energy as a function of the coverage of protons. (b) Change in Volmer reaction energy as a function of the work function. With an incomplete bilayer, $\partial \Delta^{2} E_{\text {Volmer }} / \partial \bar{\Phi}^{2}$ is equivalent to the case with no implicit solvent, indicating equivalent capacitance. However, $\partial^{2} \Delta E_{\text {Volmer }} / \partial \bar{\theta}^{2}$ is significantly reduced with the incomplete bilayer, indicating a reduced proton-proton interaction.

As we showed earlier in Note 1 , the points in the above plots are exactly equivalent to the instantaneous derivatives. That is, the points in Figure S4(a) correspond to $\partial E / \partial \theta$, while the points in Figure S4(b) correspond to $\partial E / \partial \Phi$, and therefore the slope of the lines correspond to $\partial^{2} E / \partial \theta^{2}$ and $\partial^{2} E / \partial \Phi^{2}$ respectively. Using the chain rule, we can write

$$
\frac{\partial^{2} E}{\partial \Phi^{2}}=\frac{\partial^{2} E}{\partial \theta^{2}}\left(\frac{\partial \theta}{\partial \Phi}\right)^{2}
$$

In a 6x3 supercell of Pt (111), we calculated from DFT the change in work function upon the removal of a proton from the water bilayer (i.e. via the Volmer reaction), tabulated in 
Table S1.

Table S1: Calculated changes in work function across Volmer reaction coordinate in a 6x3 supercell of Pt (111)

\begin{tabular}{cc}
\hline Case & $\Delta \Phi / \mathrm{eV}$ \\
\hline Implicit, full bilayer & 0.26 \\
Implicit, incomplete bilayer & 0.32 \\
No implicit, full bilayer & 0.79 \\
No implicit, incomplete bilayer & 0.84 \\
\hline
\end{tabular}

Because the cell size is the same for each of these cases, these numbers can be used as approximations to a number proportional to the inverse of the instantaneous proton dipole moment, $\partial \theta / \partial \Phi$,

$$
\frac{\partial \Phi}{\partial \theta} \propto \Delta \Phi
$$

Returning to Figure S4(b), we can see that the slope of the line corresponding to an incomplete bilayer is the same as that of the case with no continuum solvation, which explains the reasonable agreement in Figure S3. However, as seen in Figure S4(a) since the slope of the line corresponding to an incomplete bilayer is different than the other cases, this means that using an incomplete bilayer changes $\partial^{2} E / \partial \theta^{2}$, which represents the proton-proton interaction. In other words, while the use of an incomplete bilayer does in fact change the capacitance in a way that allows the work function to be used as the descriptor, it does so by decreasing the interaction between protons, i.e. the explicit capacitance.

Ideally, the capacitance would instead be tuned the other way, without affecting the proton-proton interaction and instead increasing the capacitance associated with continuum charging. This in principle could be done by for example changing the parameters built into the continuum model. However this would necessarily affect other factors in the system, such as the solvation energy. It would also not be easily generalizable to new systems, and would have to be fit for each case, limiting its use in practice. 


\section{Note 4}

Here we expand upon the methods used to generate the data found in Figure 7 in the main text.

In Figure S5, we show the Volmer reaction energy, with no continuum solvation, for varying water structures at each point.

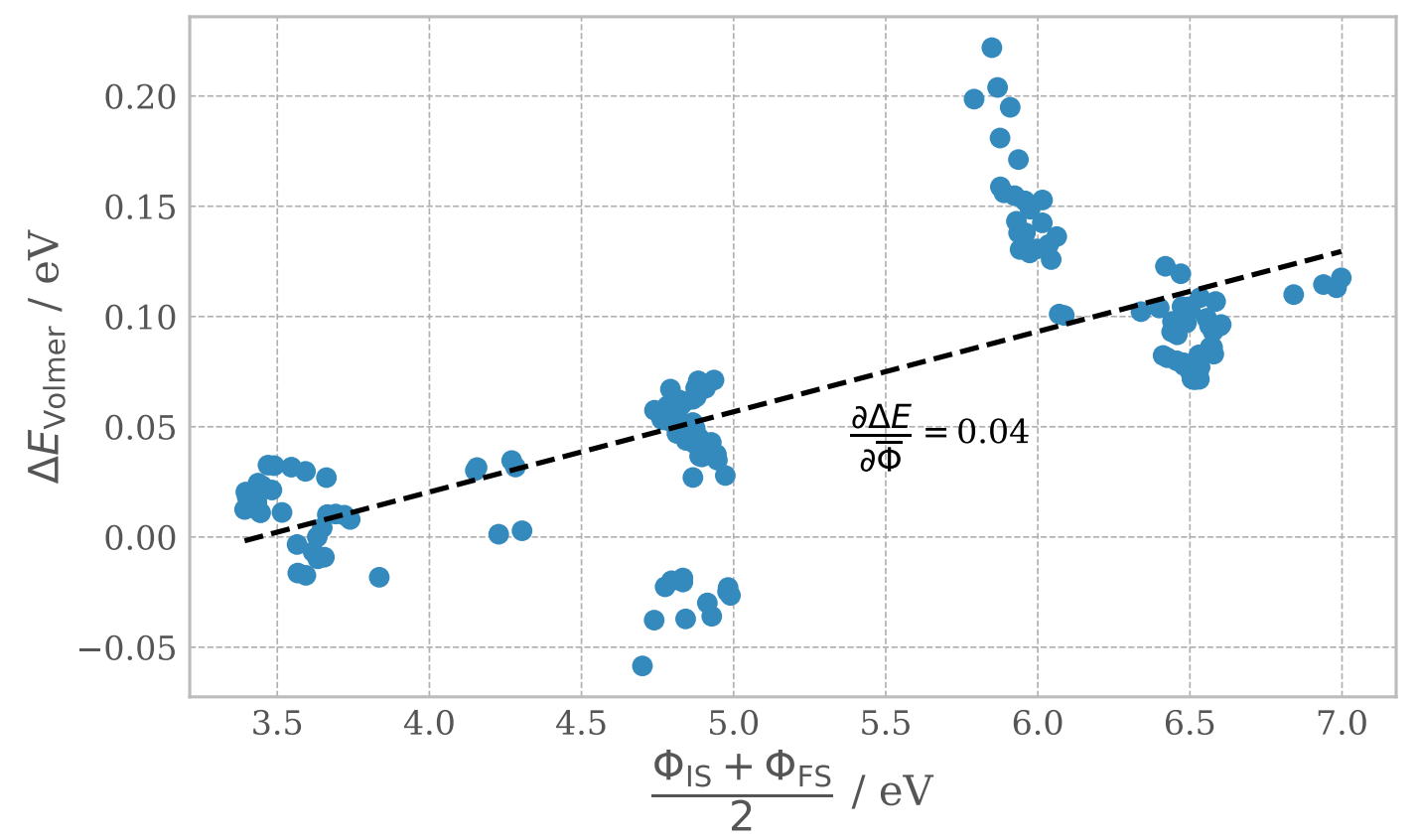

Figure S5: Volmer reaction energy as a function of work function for approximately 100 different water structures. The first water bilayer is identical for each point, while the three layers above change for each point. Despite a more than $3 \mathrm{eV}$ change in the work function, the range of Volmer reaction energies varies by less than $0.3 \mathrm{eV}$.

As can be seen in Figure S5, there is a wide range of work function and yet only a small range of energies. The small amount of scatter is likely due to very slightly different water structures between initial and final state. The picture in this system is directly analogous to the Frumkin correction, where only the potential drop between the electrode and the inner Helmholtz plane (IHP) contributes to the electron transfer driving force. Here, only the potential drop between the electrode and the first solvent bilayer contributes to the reaction driving force; the changes in measured work function convolute this potential drop and the 
potential drop due to water dipoles. This results in a huge variation of work function, but with very little change in driving force.

To generate the different water structures, the first water bilayer and the entire Pt (111) slab were fixed, and the top three water bilayers then underwent 5 picoseconds of ab-initio molecular dynamics (AIMD). A Nosé thermostat $(\mathrm{IBRION}=0$, SMASS $=3$ ) was used to control the temperature, with a set point of $300 \mathrm{~K}$, and a time-step of 1 picosecond $($ POTIM = 1.). Van der Waals interactions were accounted for using the damped DFT-D3 correction of Grimme ${ }^{2,3}$ (IVDW $\left.=11\right)$. From the MD trajectory, snapshots were taken every 20 femtoseconds. The water structure in the top three layers was relaxed above the initial and final state of the Volmer reaction, where the first bilayer was fixed to avoid chemical effects convoluting the energetics. This then gives approximately 250 Volmer reaction energies, each with a different water structure. The mean square displacement between the initial and final state were calculated illustrated in Figure S6.

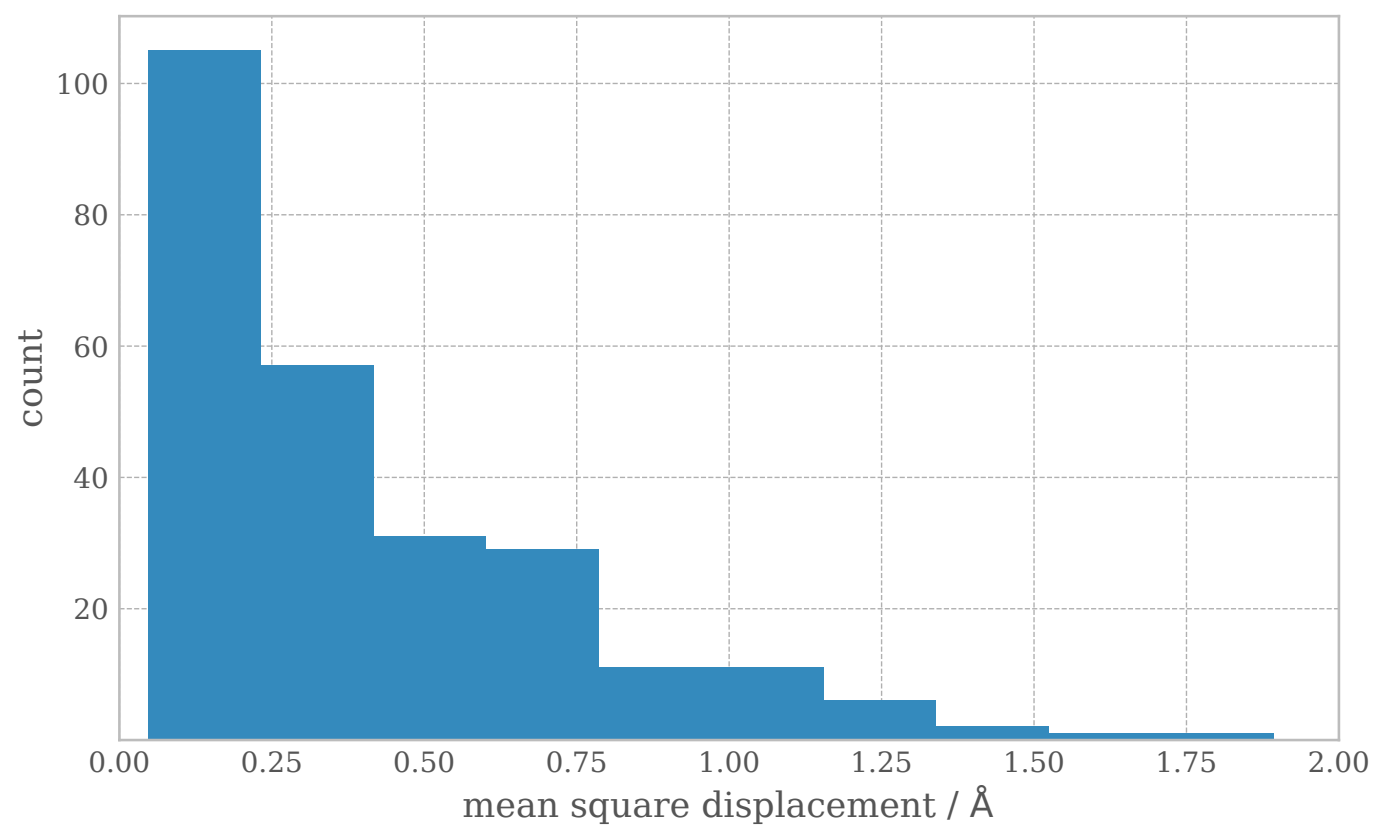

Figure S6: Histogram illustrating the mean square displacement between initial and final states for the 254 Volmer reaction energies calculated.

The purpose of this exercise is to illustrate that, when holding chemical effects (e.g. 
hydrogen bonding, solvation) constant, the contribution of the changed water structure to the driving force of the reaction is negligible. To this end we did not include the energies associated with any water structure where the initial state and final state differed by a mean square displacement of greater than $0.5 \AA$. We note that including all trajectories does not significantly change the slope of the line shown in Figure 7 in the main text, going from 0.036 to 0.047 by including every water structure. However, the range of energies (that is, the highest $\Delta E_{\text {Volmer }}$ minus the lowest $\left.\Delta E_{\text {Volmer }}\right)$ does change somewhat, illustrated in Figure S7. This can be explained by the change in water structure between initial and final state causing a change in the chemical effects we presume to be constant.

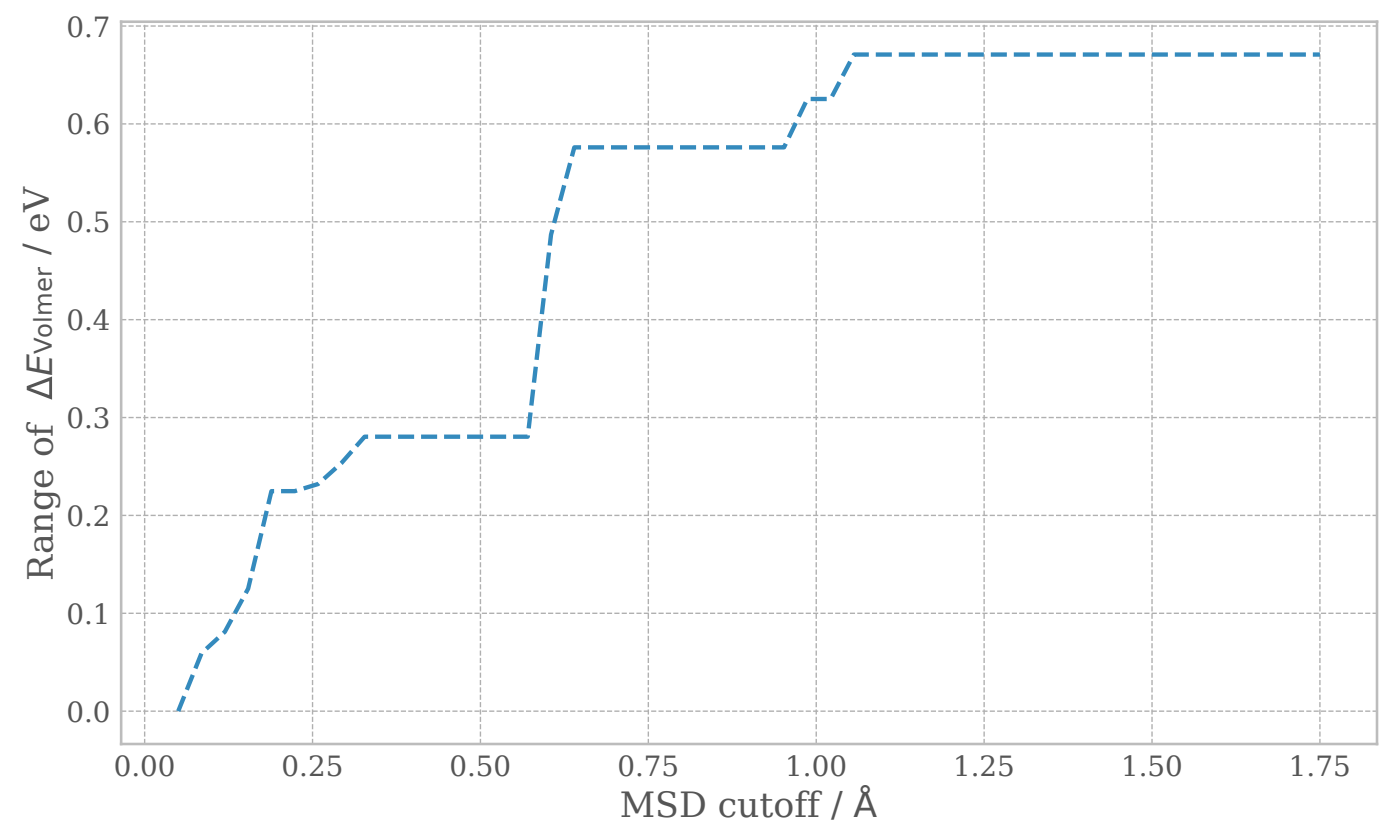

Figure S7: Range of energies (highest $\Delta E_{\text {Volmer }}$ minus lowest $\Delta E_{\text {Volmer }}$ ) as a function of the cutoff mean square displacement.

\section{Note 5}

Here we show the capacitance curves associated with our illustration that the reaction energetics are insensitive to the countercharge placement model chosen. Figure S8 illustrates that the capacitance can change somewhat significantly, which corresponds to a significant 
difference in energetics as a function of potential.

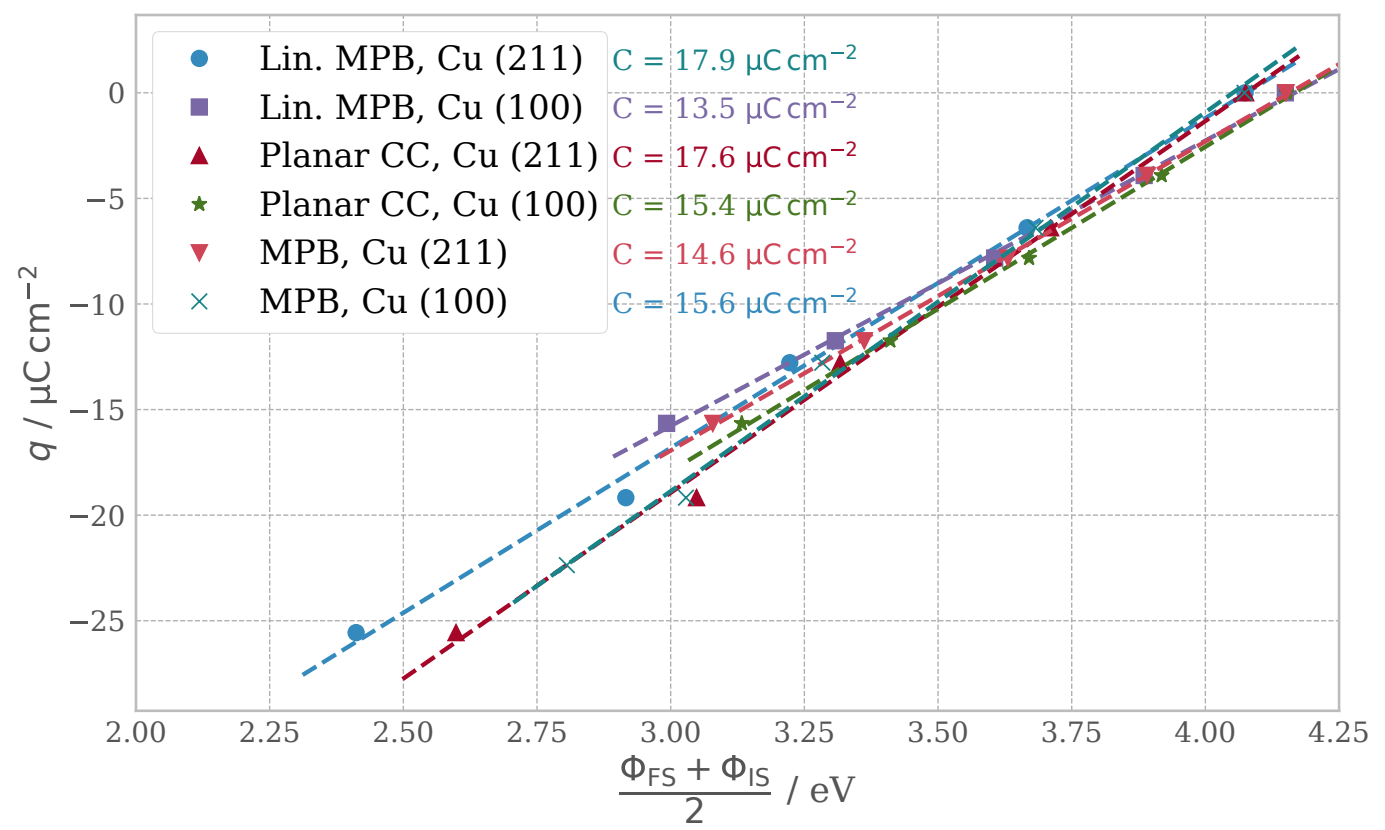

Figure S8: Capacitance curves for $\mathrm{Cu}$ (211) and $\mathrm{Cu}$ (100) with three charge placement models: the modified Poisson-Boltzmann (MPB) and its linearized version (LMPB) and a planar counter-charge (PCC).

\section{References}

(1) Mamatkulov, M.; Filhol, J.-S. An ab initio study of electrochemical vs. electromechanical properties: the case of CO adsorbed on a Pt(111) surface. Physical chemistry Chemical Physics : PCCP 2011, 13, 7675-7684.

(2) Grimme, S.; Antony, J.; Ehrlich, S.; Krieg, H. A consistent and accurate ab initio parametrization of density functional dispersion correction (DFT-D) for the 94 elements H-Pu. The Journal of Chemical Physics 2010, 132, 154104.

(3) Grimme, S.; Ehrlich, S.; Goerigk, L. Effect of the damping function in dispersion corrected density functional theory. Journal of Computational Chemistry 2011, 32, 14561465. 


\section{Tables and data corresponding to figures from the main text}

Here we provide tables and input files necessary to reproduce all figures from the main text.

\section{Figures 1-3}

The data used in the plots of Figure 1 have deliberately arbitrary units and so no values are provided. Figures 2 and 3 are similarly explanatory schematics with no data to provide.

\section{Figure 4}

Data corresponding to Figure 4:

\begin{tabular}{l|l|l}
$E / \mathrm{eV}$ & $q / \mu \mathrm{C} \mathrm{cm}^{-2}$ & $\Phi / \mathrm{eV}$ \\
\hline 3.59217955516 & -34.8284051634 & 6.54577080861 \\
2.94602229419 & -29.0236709695 & 6.37106408838 \\
1.11745081766 & -11.6094683878 & 5.79784938832 \\
0.547778509728 & -5.80473419391 & 5.58565829728 \\
0.0 & 0.0 & 5.36309412301 \\
-0.524280672303 & 5.80473419391 & 5.13279412303 \\
-1.02469150461 & 11.6094683878 & 4.89749412305 \\
-2.37164365156 & 29.0236709695 & 4.09759412312 \\
-2.76694917388 & 34.8284051634 & 3.82759412314
\end{tabular}

Geometry input (POSCAR):

Pt

1.00000000000000

5.64161579672474820 .00000000000000000 .0000000000000000

$-2.82080789836237464 .88578259835521770 .0000000000000000$

0.00000000000000000 .000000000000000016 .9312444075633444

$\mathrm{Pt}$ 
16

Selective dynamics

Direct

0.00000000000000000 .00000000000000000 .2953120207612443 T T T

0.33333333333333570 .16666666666666430 .4319843258704026 F F F

0.16666666666666430 .33333333333333570 .5680156741295903 F F F

$0.50000000000000000 .00000000000000000 .7046879792387557 \mathrm{~T}$ T T

$0.0000000000000000 \quad 0.50000000000000000 .2953120207612443$ T T T

0.33333333333333570 .66666666666666430 .4319843258704026 F F F

0.16666666666666430 .83333333333333570 .5680156741295903 F F F

$0.50000000000000000 .50000000000000000 .7046879792387557 \mathrm{~T}$ T T

$0.50000000000000000 .00000000000000000 .2953120207612443 \mathrm{~T}$ T T

0.83333333333333570 .16666666666666430 .4319843258704026 F F F

0.66666666666666430 .33333333333333570 .5680156741295903 F F F

0.00000000000000000 .00000000000000000 .7046879792387557 T T T

0.50000000000000000 .50000000000000000 .2953120207612443 T T T

0.83333333333333570 .66666666666666430 .4319843258704026 F F F

0.66666666666666430 .83333333333333570 .5680156741295903 F F F

0.00000000000000000 .50000000000000000 .7046879792387557 T T T

VASP input file (INCAR):

INCAR created by Atomic Simulation Environment

$\mathrm{ENCUT}=500.000000$

$\mathrm{SIGMA}=0.050000$

$\mathrm{EDIFF}=0.0001$

$\mathrm{EDIFFG}=-0.03$

PREC $=$ Accurate 


$$
\begin{aligned}
& \text { GGA }=\text { RP } \\
& \text { ALGO }=\text { Normal } \\
& \text { ISMEAR }=0 \\
& \text { NELM }=250 \\
& \text { NSW }=1000 \\
& \text { IBRION }=2 \\
& \text { NCORE }=16 \\
& \text { KPAR }=7 \\
& \text { LVHAR }=. \text { TRUE. } \\
& \text { POTIM }=0.2 \\
& \text { LSOL }=. \text { TRUE. } \\
& \text { LAMBDA_D_K }=3.0 \\
& \text { TAU }=0 \\
& \text { NELECT }=160.0
\end{aligned}
$$

Note that for different charges, the entry "NELECT" was varied to produce the different data points.

\section{Figure 5}

Data corresponding to Figure 5:

\begin{tabular}{l|l}
$\Delta E_{\mathrm{CO}_{2}} / \mathrm{eV}$ & $\bar{\Phi} / \mathrm{eV}$ \\
\hline 0.792935455 & 6.28890967801 \\
0.770970825 & 5.97826231432 \\
0.738200705 & 5.88380788214 \\
0.74461845 & 5.89182506158 \\
0.738964705 & 5.83729891305 \\
0.75026978 & 5.79672813601
\end{tabular}




\begin{tabular}{l|l}
0.726125725 & 5.7424552028 \\
0.70478197 & 5.69967657212 \\
0.72697721 & 5.69418021333
\end{tabular}

\begin{tabular}{l|l}
$\Delta E_{\text {Volmer }} / \mathrm{eV}$ & $\bar{\Phi} / \mathrm{eV}$ \\
\hline 0.007504865 & 4.89874704757 \\
0.007504865 & 4.89874704757 \\
0.41209548 & 5.40688860091 \\
0.41209548 & 5.40688860091 \\
-0.49698839 & 4.45877079266 \\
0.61101113 & 5.71845917931 \\
-0.569108955 & 4.25263910041 \\
0.72221029 & 6.05602074766 \\
-0.6297205 & 4.0919129468 \\
0.865047195 & 6.2688255888
\end{tabular}

INCAR file used for all relaxations:

INCAR created by Atomic Simulation Environment

$\mathrm{ENCUT}=500.000000$

$\mathrm{SIGMA}=0.050000$

$\mathrm{EDIFF}=1.00 \mathrm{e}-04$

$\mathrm{EDIFFG}=-3.00 \mathrm{e}-02$

$\mathrm{PREC}=$ Accurate

$\mathrm{GGA}=\mathrm{RP}$

ALGO $=$ Normal

$\mathrm{ISMEAR}=0$ 

$\mathrm{NELM}=250$
IBRION $=2$
$\mathrm{NSW}=1000$
$\mathrm{NCORE}=8$
LCHARG $=$.FALSE.
LVHAR $=$.TRUE.
POTIM $=0.2$

\section{Figure 6}

Data corresponding to Figure 6:

\begin{tabular}{l|l|l}
$\Delta E_{\mathrm{CO}_{2}} / \mathrm{eV}$ & $\bar{\Phi} / \mathrm{eV}$ & $\frac{\overline{q_{\mathrm{exp}}}+\overline{q_{\mathrm{imp}}}}{A} / \mu \mathrm{Ccm}^{-2}$ \\
\hline 0.828276295904 & 6.49583282309 & -18.98422594965748 \\
0.759159431139 & 6.14206438771 & -13.187515736021604 \\
0.68698319 & 5.73174670785 & -7.390805522385735 \\
0.609809068873 & 5.28659732377 & -1.5940953087498642 \\
0.527712109267 & 4.80426334468 & 4.202614904886007 \\
0.662830706404 & 5.63750003294 & -4.218716877701725 \\
0.64629998524 & 5.54816033602 & -2.93055905244929 \\
0.63054565 & 5.4486691568 & -1.6424012271968547 \\
0.613580105537 & 5.35092466013 & -0.3542434019444195 \\
0.596162021355 & 5.25031003708 & 0.9339144233080157 \\
0.655021204929 & 5.66675165168 & -4.752626121260275 \\
0.637691037627 & 5.5608497206 & -3.3014425727838552 \\
0.61988995 & 5.45249300547 & -1.850259024307435 \\
0.601384484698 & 5.34162941458 & -0.3990754758310153 \\
0.5823098632 & 5.22829286981 & 1.0521080726454044 \\
0.628624848854 & 5.56851868627 & -3.164037658276294
\end{tabular}




\begin{tabular}{|c|c|c|}
\hline 0.616748694948 & 5.49756931374 & -2.1979192893369675 \\
\hline 0.604670905 & 5.42467993696 & -1.2318009203976412 \\
\hline 0.592208413022 & 5.35143035821 & -0.2656825514583149 \\
\hline .579540496008 & 5.2759801734 & 0.7004358174810115 \\
\hline 0.622193209356 & 5.50065365697 & -2.1093584388508626 \\
\hline 0.610934092943 & 5.45866764064 & -1.465279526224645 \\
\hline 0.603850005 & 5.40769888684 & -0.8212006135984273 \\
\hline 0.595544993331 & 5.3583025981 & -0.17712170097220975 \\
\hline 0.587245114004 & 5.30774025542 & 0.46695721165400783 \\
\hline 0.671657481053 & 5.75010709701 & -6.328075316552487 \\
\hline 0.646179401271 & 5.6041674754 & -4.395838578673864 \\
\hline 0.621835495 & 5.47060795729 & -2.4636018407952425 \\
\hline 0.591609087227 & 5.32983210385 & -0.5313651029166206 \\
\hline 0.56354714075 & 5.1830535637 & 1.4008716349620012 \\
\hline 0.593897902674 & 5.6049490798 & -3.996679147296306 \\
\hline 0.57678212967 & 5.51764258813 & -2.7763191023203344 \\
\hline 0.56015639 & 5.43087294754 & -1.5559590573443633 \\
\hline 0.543429494099 & 5.34125882391 & -0.33559901236839207 \\
\hline 0.526641696634 & 5.25017264956 & 0.8847610326075792 \\
\hline 0.605771838073 & 5.52015123547 & -2.8124779184677715 \\
\hline 0.592672531753 & 5.45641443438 & -1.9537060349661615 \\
\hline 0.58310706 & 5.39182693452 & -1.094934151464552 \\
\hline 0.566062275784 & 5.32740140522 & -0.23616226796294246 \\
\hline 0.55243641026 & 5.26183996155 & 0.6226096155386671 \\
\hline 0.689813625067 & 6.01435067712 & -10.848129114089977 \\
\hline 0.646357171949 & 5.79531694574 & -7.535723277726625 \\
\hline 0.603153745 & 5.56737224341 & -4.223317441363274 \\
\hline
\end{tabular}


\begin{tabular}{l|l|l}
0.558102688292 & 5.34260847179 & -0.9109116049999221 \\
0.514959956987 & 5.07329868242 & 2.4014942313634298
\end{tabular}

\begin{tabular}{|c|c|c|}
\hline$\Delta E_{\text {Volmer }} / \mathrm{eV}$ & $\bar{\Phi} / \mathrm{eV}$ & $\frac{\overline{q_{\exp }}+\overline{q_{\text {imp }}}}{A} / \mu \mathrm{C} \mathrm{cm}^{-2}$ \\
\hline 0.413841084168 & 5.45765 & 5.8266235333340495 \\
\hline 0.232051412662 & 4.9584 & 7.3400322432909455 \\
\hline 0.0432996742747 & 4.3851 & 8.853440953247842 \\
\hline-0.1410884464 & 3.7734 & 10.36684966320474 \\
\hline-0.31470211713 & 3.1619 & 11.880258373161636 \\
\hline 0.906838522791 & 5.63425 & 2.9133117666670247 \\
\hline 0.802680585854 & 5.34565 & 3.6700161216454728 \\
\hline 0.691814566293 & 5.0264 & 4.426720476623921 \\
\hline 0.577374433356 & 4.68915 & 5.18342483160237 \\
\hline 0.461897614678 & 4.34555 & 5.940129186580818 \\
\hline 1.10503628531 & 5.8505 & 1.4566558833335124 \\
\hline 1.05101780837 & 5.70375 & 1.8350080608227364 \\
\hline 0.995422465683 & 5.5461 & 2.2133602383119606 \\
\hline 0.937583068087 & 5.3818 & 2.591712415801185 \\
\hline 0.889163911859 & 5.2121 & 2.970064593290409 \\
\hline 1.00885672648 & 5.7751 & 1.942207844444683 \\
\hline 0.941590998984 & 5.5837 & 2.4466774144303156 \\
\hline 0.870267713821 & 5.37485 & 2.9511469844159475 \\
\hline 0.79601573533 & 5.1519 & 3.4556165544015793 \\
\hline 0.719870898858 & 4.92015 & 3.960086124387212 \\
\hline 0.413839689583 & 5.4577 & 5.8266235333340495 \\
\hline 0.232049303777 & 4.95845 & 7.3400322432909455 \\
\hline 0.0433002185036 & 4.3851 & 8.853440953247842 \\
\hline
\end{tabular}




\begin{tabular}{l|l|l}
-0.14108569125 & 3.77335 & 10.36684966320474 \\
-0.31470051846 & 3.16185 & 11.880258373161636 \\
-0.310761602446 & 4.54255 & 11.766752719914868 \\
-0.41748400834 & 4.2351 & 12.523457074893317 \\
-0.519474082263 & 3.92145 & 13.280161429871765 \\
-0.615820480911 & 3.6061 & 14.03686578485021 \\
-0.706223504891 & 3.2947 & 14.793570139828661 \\
-0.64176007385 & 3.91245 & 14.736817313205277 \\
-0.683218761871 & 3.76175 & 15.115169490694502 \\
-0.723228851806 & 3.61175 & 15.493521668183725 \\
-0.761750274913 & 3.46165 & 15.871873845672948 \\
-0.798805276449 & 3.3118 & 16.250226023162174 \\
-0.514855119713 & 4.1214 & 13.746795782108473 \\
-0.577191300734 & 3.91815 & 14.251265352094103 \\
-0.63700702721 & 3.714 & 14.755734922079737 \\
-0.69419852172 & 3.51005 & 15.26020449206537 \\
-0.748686769221 & 3.3074 & 15.764674062051002 \\
\hline
\end{tabular}

\section{Figure 7}

POSCAR file corresponding to high work function case:

Pt H O

1.00000000000000

8.46383999999999940 .00000000000000000 .0000000000000000

0.00000000000000004 .88659999999999960 .0000000000000000

0.00000000000000000 .000000000000000032 .1040899999999993

Pt H O 
183316

Selective dynamics

Direct

0.00000000000000000 .08333333000000210 .2180407600000009 F F F 0.33333333000000210 .08333333000000210 .2180407600000009 F F F 0.66666666999999790 .08333333000000210 .2180407600000009 F F F 0.16666666999999790 .58333333000000210 .2180407600000009 F F F 0.50000000000000000 .58333333000000210 .2180407600000009 F F F 0.83333333000000210 .58333333000000210 .2180407600000009 F F F 0.16666666999999790 .25000000000000000 .2897937799999966 F F F 0.50000000000000000 .25000000000000000 .2897937799999966 F F F 0.83333333000000210 .25000000000000000 .2897937799999966 F F F 0.33333333000000210 .75000000000000000 .2897937799999966 F F F 0.66666666999999790 .75000000000000000 .2897937799999966 F F F 0.00000000000000000 .75000000000000000 .2897937799999966 F F F 0.99859277000000190 .42208226000000340 .3635304200000036 F F F 0.33217814000000350 .42152286000000320 .3622850900000003 F F F 0.66581560000000200 .42044240999999970 .3622405099999995 F F F 0.16628784000000250 .92205889000000240 .3627106799999993 F F F 0.49959188999999780 .92046506999999880 .3628199999999993 F F F 0.83285820999999770 .92096839999999960 .3623438099999987 F F F 0.02944313999999790 .26917026000000280 .4674948400000005 F F F 0.96530964000000100 .45416620000000310 .4291165899999996 F F F 0.83692841999999960 .42851944000000230 .4730057299999970 F F F 0.42738826000000070 .94383080000000060 .4723800299999965 F F F 0.54325038999999720 .93450166000000220 .4348752300000029 F F F 0.10403230000000010 .82821375000000330 .4717710199999985 F F F 
0.17368161000000270 .99565798999999800 .4352335800000020 F F F 0.63136595999999660 .26644345999999790 .4787605400000032 F F F 0.63265634999999780 .59684013999999760 .4790003100000035 F F F 0.22304241648048870 .81746716873205120 .6253580176131806 T T T 0.21830872018083620 .13088337159069850 .6179862456189582 T T T 0.78036007670294080 .83282372589255260 .5926057183335232 T T T 0.83792286738900400 .80035225855120020 .5469894285181809 T T T 0.61933008194664300 .77310537397739410 .6518156274972142 T T T 0.50518573008069010 .89945171151817500 .6193574696520372 T T T 0.99235912130156120 .16792557840630450 .5807113540913349 T T T 0.00121518763704390 .48561325710323190 .5849655036965515 T T T 0.88377315038921010 .14403397831549110 .7891641525153545 T T T 0.83238182137539950 .23037339690912970 .7448038184587702 T T T 0.30556258197269410 .80018255469978070 .7324575755137417 T T T 0.35372197503330940 .92520669064544590 .6899294023110443 T T T 0.05666009161902250 .52181828587814040 .7571973231186533 T T T $0.04670661573642580 .84489471860228350 .7572548530839995 \mathrm{~T}$ T T 0.52206212829010920 .20497220659951410 .7331325798659449 T T T 0.53091984416594330 .52104875205780130 .7260655694049092 T T T 0.18004949472393860 .93660931471387700 .8669044249580651 T T T 0.01723185837778370 .08834631015931650 .8663441918502812 T T T 0.48909329598930640 .56272924385301340 .8362092637478753 T T T 0.59421984522423800 .49723625043336030 .7977762235164718 T T T 0.22169587434200370 .42055129795808450 .8539078144155710 T T T 0.22183187432702310 .61980769506131140 .8156941944582599 T T T 0.72756113483467290 .89342739805245230 .8364787604590234 T T T 0.71826412667054740 .21372214520989980 .8418962785541098 T T T 
0.96550092999999750 .44081727999999740 .4616855200000032 F F F 0.54092363000000180 .93617766999999930 .4662140300000033 F F F 0.15832471000000230 .00160566000000270 .4663059100000027 F F F 0.69248180000000300 .43099134999999930 .4876129100000028 F F F $0.28419261690035570 .98353609383728010 .6300662091402671 \mathrm{~T}$ T T $0.87763018534828060 .83200653481329570 .5750033418506320 \mathrm{~T}$ T T 0.60899068796881290 .81075220965256990 .6221956843579832 T T T 0.06708538808005220 .32023191454513980 .5848475065411236 T T T 0.92730176583107490 .18462268435521880 .7608212833321062 T T T $0.39909554408661310 .86286593923839660 .7164027856266770 \mathrm{~T}$ T T 0.12137187308487540 .68991008322590370 .7579958131191589 T T T $0.59223657792534110 .36430794060868270 .7362794802111381 \mathrm{~T}$ T T 0.13010991560044260 .11585114002743070 .8714235397231249 T T T 0.60125728123923490 .54792019709189080 .8273101221137296 T T T 0.27088270209426210 .59218663616333340 .8430625635146285 T T T $0.79247584791547610 .06090262897693320 .8373697223640235 \mathrm{~T}$ T T

INCAR file corresponding to high work function case:

INCAR created by Atomic Simulation Environment

$\mathrm{ENCUT}=500.000000$

SIGMA $=0.050000$

$\mathrm{EDIFF}=1.00 \mathrm{e}-04$

$\mathrm{EDIFFG}=-5.00 \mathrm{e}-02$

$\mathrm{PREC}=$ Accurate

$\mathrm{GGA}=\mathrm{RP}$

$\mathrm{ALGO}=$ Normal

$\operatorname{ISMEAR}=0$ 

$\mathrm{NELM}=250$
IBRION $=2$
$\mathrm{NSW}=0$
$\mathrm{NCORE}=16$
$\mathrm{KPAR}=14$
LWAVE $=$.FALSE.
LVHAR $=$.TRUE.
LDIPOL $=$.TRUE.
$\mathrm{IDIPOL}=3$
$\mathrm{DIPOL}=0.50 .50 .5$

POSCAR file corresponding to low work function case:

\section{Pt H O}

1.00000000000000

8.46383999999999940 .00000000000000000 .0000000000000000

0.00000000000000004 .88659999999999960 .0000000000000000

0.00000000000000000 .000000000000000032 .1040899999999993

\section{$\mathrm{Pt} \mathrm{H} \mathrm{O}$}

183316

Selective dynamics

Direct

0.00000000000000000 .08333333000000210 .2180407600000009 F F F 0.33333333000000210 .08333333000000210 .2180407600000009 F F F 0.66666666999999790 .08333333000000210 .2180407600000009 F F F 0.16666666999999790 .58333333000000210 .2180407600000009 F F F 0.50000000000000000 .58333333000000210 .2180407600000009 F F F 0.83333333000000210 .58333333000000210 .2180407600000009 F F F 
0.16666666999999790 .25000000000000000 .2897937799999966 F F F 0.50000000000000000 .25000000000000000 .2897937799999966 F F F 0.83333333000000210 .25000000000000000 .2897937799999966 F F F 0.33333333000000210 .75000000000000000 .2897937799999966 F F F 0.66666666999999790 .75000000000000000 .2897937799999966 F F F 0.00000000000000000 .75000000000000000 .2897937799999966 F F F 0.99859277000000190 .42208226000000340 .3635304200000036 F F F 0.33217814000000350 .42152286000000320 .3622850900000003 F F F 0.66581560000000200 .42044240999999970 .3622405099999995 F F F 0.16628784000000250 .92205889000000240 .3627106799999993 F F F 0.49959188999999780 .92046506999999880 .3628199999999993 F F F 0.83285820999999770 .92096839999999960 .3623438099999987 F F F 0.02944313999999790 .26917026000000280 .4674948400000005 F F F 0.96530964000000100 .45416620000000310 .4291165899999996 F F F 0.83692841999999960 .42851944000000230 .4730057299999970 F F F 0.42738826000000070 .94383080000000060 .4723800299999965 F F F 0.54325038999999720 .93450166000000220 .4348752300000029 F F F 0.10403230000000010 .82821375000000330 .4717710199999985 F F F 0.17368161000000270 .99565798999999800 .4352335800000020 F F F 0.63136595999999660 .26644345999999790 .4787605400000032 F F F 0.63265634999999780 .59684013999999760 .4790003100000035 F F F $0.04735690911474680 .53383701791831580 .5809194215471081 \mathrm{~T}$ T T 0.18898868448962960 .52978580831914000 .6115497494065565 T T T 0.54300350231886800 .01687405050879680 .5703461172756690 T T T $0.70864729683903250 .16286690897155860 .5765965883073321 \mathrm{~T}$ T T 0.27816193221769940 .20632042496922050 .5624837339490796 T T T 0.27264182742649010 .88492835357981650 .5645740414386466 T T T 
0.81334079591591290 .49011592089669880 .6167188784944742 T T T $0.77341292570309860 .66181549074815390 .5775421773971701 \mathrm{~T}$ T T 0.08011442212470850 .42137096752593100 .7255804654487576 T T T 0.96562283866573040 .43994051955637300 .6872411315744600 T T T $0.47652236876746910 .95375727644908890 .6734924166530121 \mathrm{~T}$ T T $0.60054538857770720 .97473213600029850 .6365255872375783 \mathrm{~T}$ T T 0.18841204891464970 .76080322497981710 .6884832064667776 T T T 0.18655797001907360 .08522811808800410 .6874104264436767 T T T 0.69276870481799340 .30157806839086730 .6759640971063590 T T T 0.69614594194793970 .62933421311949190 .6764728744331734 T T T 0.15509369271263300 .22681864087506650 .7863895138082455 T T T 0.99274998360186120 .38529501733759730 .7936940419594123 T T T 0.50457758062417920 .87198491225239390 .7918430751324621 T T T $0.66512764873191800 .03170885318396440 .7984368915495850 \mathrm{~T}$ T T 0.22389198378679960 .74062387010066290 .7853587889874376 T T T 0.28246633878002570 .91537291617001420 .7480500081139922 T T T 0.77831419304493470 .37599596978324000 .8428437513460239 T T T $0.72015802493240950 .55013254815749950 .8052429406554751 \mathrm{~T}$ T T 0.96550092999999750 .44081727999999740 .4616855200000032 F F F 0.54092363000000180 .93617766999999930 .4662140300000033 F F F 0.15832471000000230 .00160566000000270 .4663059100000027 F F F 0.69248180000000300 .43099134999999930 .4876129100000028 F F F 0.16496034785949120 .54295889083755360 .5819721948590839 T T T 0.64999067755934450 .99362306209640390 .5828083419479597 T T T 0.34221482531657440 .04079061131717050 .5586193553233656 T T T 0.83476226696634370 .50003836986321200 .5864408347831258 T T T 0.07956085807054340 .43011057212019210 .6946597630459124 T T T 
0.59038978523887660 .96640096356976100 .6675225078555442 T T T 0.25617441711528950 .92508639522542070 .6915133162554596 T T T 0.76219034295367290 .46356962626649080 .6729873885398518 T T T 0.10048750514356190 .40374653370824380 .7821832798600923 T T T 0.61840069213806000 .84818705345379900 .7973289111624737 T T T $0.2831748126168350 \quad 0.90932878427279460 .7790138576754799$ T T T 0.78011339923935450 .37941084802574920 .8125640314776490 T T T

INCAR file corresponding to low work function case:

INCAR created by Atomic Simulation Environment

$\mathrm{ENCUT}=500.000000$

$\mathrm{SIGMA}=0.050000$

$\mathrm{EDIFF}=1.00 \mathrm{e}-04$

$\mathrm{EDIFFG}=-5.00 \mathrm{e}-02$

PREC $=$ Accurate

$\mathrm{GGA}=\mathrm{RP}$

$\mathrm{ALGO}=$ Normal

$\operatorname{ISMEAR}=0$

$\mathrm{NELM}=250$

IBRION $=2$

$\mathrm{NSW}=0$

NCORE $=16$

$\mathrm{KPAR}=14$

LWAVE $=$.FALSE.

LVHAR $=$.TRUE.

$\mathrm{LDIPOL}=$.TRUE.

IDIPOL $=3$ 
$\mathrm{DIPOL}=0.50 .50 .5$

\section{Figure 8}

Data corresponding to Figure 8:

\begin{tabular}{l|l|l|l}
$2 \Delta E_{\mathrm{CO}}$ (Lin. Mod. PB) / eV & Facet & $q / \mu \mathrm{C} \mathrm{cm}^{-2}$ & $\bar{\Phi} / \mathrm{eV}$ \\
\hline-1.4563549651975336 & 211 & -25.563257651181587 & 2.4116999999999997 \\
-1.3756121224760136 & 211 & -19.17244323838619 & 2.9161 \\
-1.3285761570114119 & 211 & -12.781628825590793 & 3.2227 \\
-1.3215146668408124 & 211 & -6.390814412795397 & 3.66655 \\
-1.3133663539992995 & 211 & 0.0 & 4.07425 \\
-1.1604698058217764 & 100 & -15.654234352173216 & 2.99235 \\
-1.119176802916627 & 100 & -11.740675764129913 & 3.30785 \\
-1.0760934950449155 & 100 & -7.827117176086608 & 3.6059 \\
-1.034064628292981 & 100 & -3.913558588043304 & 3.88545 \\
-0.9888406688842224 & 100 & 0.0 & 4.1495
\end{tabular}

\begin{tabular}{l|l|l|l}
$2 \Delta E_{\mathrm{CO}}$ (Planar CC) $/ \mathrm{eV}$ & Facet & $q / \mu \mathrm{C} \mathrm{cm}^{-2}$ & $\bar{\Phi} / \mathrm{eV}$ \\
\hline-1.4557301237909996 & 211 & -25.563257651181587 & 2.5985500000000004 \\
-1.3760662804706953 & 211 & -19.17244323838619 & 3.04835 \\
-1.32693966438881919 & 211 & -12.781628825590793 & 3.3169 \\
-1.3205175737093668 & 211 & -6.390814412795397 & 3.7104 \\
-1.313004374565935 & 211 & 0.0 & 4.0753 \\
-1.1610345100634731 & 100 & -15.654234352173216 & 3.1329000000000002 \\
-1.122760542173637 & 100 & -11.740675764129913 & 3.41075 \\
-1.0815117577731144 & 100 & -7.827117176086608 & 3.6702 \\
-1.037887623642746 & 100 & -3.913558588043304 & 3.91815 \\
-0.9956928354804404 & 100 & 0.0 & 4.15185
\end{tabular}




\begin{tabular}{l|l|l|l}
$2 \Delta E_{\mathrm{CO}}$ (Mod. PB) $/ \mathrm{eV}$ & Facet & $q / \mu \mathrm{C} \mathrm{cm}^{-2}$ & $\Phi / \mathrm{eV}$ \\
\hline-1.1557542090595234 & 100 & -15.654234352173216 & 3.07875 \\
-1.1162746408081148 & 100 & -11.740675764129913 & 3.3621499999999997 \\
-1.075886484439252 & 100 & -7.827117176086608 & 3.63065 \\
-1.03174220470828 & 100 & -3.913558588043304 & 3.89095 \\
-0.9893668690201594 & 100 & 0.0 & 4.15075 \\
-1.4118651689204853 & 211 & -22.367850444783887 & 2.8058 \\
-1.3731766355922446 & 211 & -19.17244323838619 & 3.02855 \\
-1.3273621211192221 & 211 & -12.781628825590793 & 3.28355 \\
-1.3215508579851303 & 211 & -6.390814412795397 & 3.68225 \\
-1.3137836405694543 & 211 & 0.0 & 4.0732
\end{tabular}


